\title{
Dynamic simulation of an electrorheological fluid
}

\author{
R. T. Bonnecaze a) and J. F. Brady \\ Department of Chemical Engineering, California Institute of Technology, Pasadena, California 91125
}

(Received 20 May 1991; accepted 23 October 1991)

\begin{abstract}
A molecular-dynamics-like method is presented for the simulation of a suspension of dielectric particles in a nonconductive solvent forming an electrorheological fluid. The method accurately accounts for both hydrodynamic and electrostatic interparticle interactions from dilute volume fractions to closest packing for simultaneous shear and electric fields. The hydrodynamic interactions and rheology are determined with the Stokesian dynamics methodology, while the electrostatic interactions, in particular, the conservative electrostatic interparticle forces, are determined from the electrostatic energy of the suspension. The energy of the suspension is computed from the induced particle dipoles by a method previously developed [R. T. Bonnecaze and J. F. Brady, Proc. R. Soc. London, Ser. A 430, 285 (1990)]. Using the simulation, the dynamics can be directly correlated to the observed macroscopic rheology of the suspension for a range of the so-called Mason number, $M a$, the ratio of viscous to electrostatic forces. The simulation is specifically applied to a monolayer of spherical particles of areal fraction 0.4 with a particle-to-fluid dielectric constant ratio of 4 for $M a=10^{-4}$ to $\infty$. The effective viscosity of the suspension increases as $M a^{-1}$ or with the square of the electric field for small $M a$ and has a plateau value at large $M a$, as is observed experimentally. This rheological behavior can be interpreted as Bingham plastic-like with a dynamic yield stress. The first normal stress difference is negative, and its magnitude increases as $M a^{-1}$ at small $M a$ with a large $M a$ plateau value of zero. In addition to the time averages of the rheology, the time traces of the viscosities are presented along with selected "snapshots" of the suspension microstructure. In particular, at small $M a$, the suspension dynamics exhibit two distinct motions: a slow elastic-body-like deformation where electrostatic energy is stored, followed by a rapid microstructural rearrangement where energy is viscously dissipated. It is suggested that the observed dynamic yield stress is associated with these dynamics.
\end{abstract}

\section{INTRODUCTION}

In the late 1930's Winslow,' an intrepid basement experimentalist, observed interesting phenomena when dielectric particles suspended in oil were subject to an electric field. He saw the electrically induced formation of fibrous particle chains aligned with the electric field and, of more interest, Winslow found that the effective viscosity of the suspension could be varied by orders of magnitude by varying the applied electric field. In fact he observed that the viscosity increased with the square of the applied electric field. This electrorheological (ER) response is often referred to as the Winslow effect.

Winslow ${ }^{2}$ recognized the potential of these new fluids with their "tunable" viscosities and patented several electromechanical devices such as clutches, brakes, and valves that could be controlled by varying the electric field. Although none of his machines were ever used commercially, there is now a renewed interest in designing controllable machines and devices using ER fluids, probably partially fueled by the electronics technology available today to quickly access and analyze system data. Of course, the devices of Winslow are only a partial list of the possibilities, but even so it is apparent that these fluids could profoundly change many aspects of present technology and industry.

\footnotetext{
" Current address: Institute of Theoretical Geophysics, Department of Applied Mathematics and Theoretical Physics, Univcrsity of Cambridge, Cambridge, England CB3 9EW.
}

Currently available ER fluids, however, do not have the necessary properties for practical application. For example, they tend to settle out of suspension, have poor tribological properties, and, most importantly, do not have a high enough range of effective viscosity (or yield stress) for practical applications. Particularly for this latter problem, we must understand the underlying physics of the ER response in order to engineer better fluids. A detailed micromechanical model relating the bulk rheological properties to the suspension microstructure would be an excellent tool for this task.

In this paper we shall endeavor to understand ER fluids with a molecular-dynamics-like simulation. We develop the methodology for the simulation of dielectric particles forming an electrorheological fluid. The simulation accurately accounts for both hydrodynamic forces due to an imposed shear flow and electrostatic forces due to the applied electric field. The simulation allows the observation of the timeevolved motions of the suspended particles and the instantaneous rheology of the suspension, so we can directly relate the suspension bulk properties to its microstructure and gain insight into the processes involved. In addition, the simulation method provides a means to test theories that describe ER suspensions, including constitutive models. But before modeling these fluids, we must have a clear idea of their characteristics.

Typical suspended materials in an ER fluid are 1-100 $\mu \mathrm{m}$ in size, approximately spherical, particles of cornstarch, 
silica, or even zeolites, while the suspending fluids are nonconducting solvents such as silica oil, chlorohydrocarbons, or corn oil. To minimize settling problems the fluids are blended so that the particles are close to neutrally buoyant, with densities in the range of $0.6-2.0 \mathrm{~g} / \mathrm{cm}^{3}$. The viscosities of the suspending fluids, at room temperature, are from 0.01-10 Pa s. The zero-field viscosity of the suspension with particle loadings ranging from $0.05-0.50$ by volume fraction are up to an order of magnitude greater. The effective viscosity of the ER suspension can be 100000 times greater for electric field strengths of about $1 \mathrm{kV} / \mathrm{mm}$ perpendicular to the direction of flow. The dielectric constants relative to vacuum for the suspending fluid vary from $2-15$ and those of the particles from $2-40$. The particle-to-fluid dielectric constant ratio typically varies from $2-10$. The particle Reynolds number is less than 0.1 (maximum shear rates are about 0.1 $\mathrm{sec}^{-1}$ ) and the fluid Reynolds number is small enough so inertia is negligible. Moreover, the particle Peclét number is extremely large, usually in excess of 100000 , so thermal or Brownian forces are negligible. Indeed the thermal forces must be small otherwise the Brownian motion would disrupt the chains of particles that are important, as we shall see, to the ER response.

The key to observing the ER response as noted by Winslow ${ }^{2}$ is the induced electrostatic polarization forces due to the dielectric mismatch between the particles and the fluid. When an uncharged particle is placed in an electric field, it develops an induced dipole if the surrounding medium has a different dielectric constant due to the differing polarizability of the two materials. This dipole is further enhanced by the presence of other particles since they intensify the local electric field experienced by any one particle. The dipole, of course, can be thought of as a concentration of positive charge at one end of the particle and negative charge at the other end. With this simple picture and using the interactions of two particles as a paradigm, we can qualitatively explain why ER fluids form chains of particles aligned with the electric field.

Let us imagine two particles whose line of centers is parallel to the electric field, such as those in Fig. 1. The force between point charges scales as $O\left(q_{\alpha} q_{\beta} / r^{2}\right)$, so there is at-

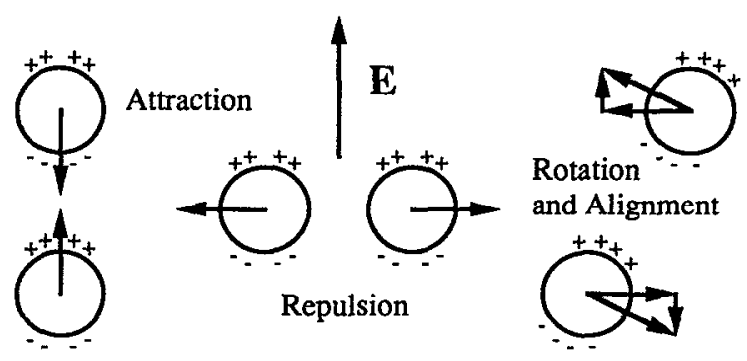

FIG. 1. Mechanism of fibration and alignment of dielectric particles. Due to the dielectric mismatch between the particles and the fluid, the particles have an induced dipole as illustrated. The interaction of these dipoles cause attraction, repulsion, rotation, and alignment of particles, creating chains that align with the applied electric field. traction between the positive and negative charges at the surfaces nearest and furthest from each other and repulsion between charges of the same sign. There is then a net attraction of the particles along their line of centers so that they move toward each other. If we apply the same considerations to particles whose line of centers is perpendicular to electric field, we find there is a net repulsion moving the particles away from each other along their line of centers. For particles whose line of centers is neither parallel nor perpendicular to the electric field, the particles may expcrience both attraction and repulsion causing the pair to eventually translate and rotate into alignment with the electric field. Thus, the forces due to polarization interactions cause the formation of particle chains aligned with the electric field. As we shall see, the microstructure of a suspension is intimately related to its bulk rheological properties, so the formation and strength of these chains strongly affect the viscosity of an ER fluid.

The microstructure and rheology of ferrofluids developed by Rosensweig ${ }^{3}$ also depend upon an applied field. However, there are fundamental differences between ferrofluids and ER fluids. A ferrofluid is a suspension of permanently magnetized particles, and so there are polarization forces without an applied magnetic field. The particles are dense and suspensions would then settle out because of particle clumping, and so the particles are usually sufficiently small such that Brownian forces, along with short-range steric repulsion, dominate in the zero-field limit to disperse the particles. As mentioned, Brownian forces are usually negligible for an ER suspension. Since the magnetic dipoles are permanent in a ferrofluid, the rheological properties scale linearly with the applied magnetic field at high field strengths in contrast to the quadratic dependence on the field strength observed in ER fiuids since their particle dipoles are induced. There is also a torque on a ferrofluid particle given by the cross product of its dipole and the applied field which gives rise to an antisymmetric stress tensor as shown by Batchelor. ${ }^{4}$ In an ER fluid of nearly spherical particles, the local electric field is aligned with the induced dipole, ${ }^{5}$ so there is no net torque on the particle. There are antisymmetric stresses in ER fluids, however, but they exist because of the "effective torque" exerted on the deformed or strained chains of particles.

Two decades after Winslow ${ }^{2}$ published his observations, an extensive study of ER fluids was performed by Klass and Martinek ${ }^{6.7}$ and Uejima. ${ }^{8}$ They both confirmed many findings of Winslow and performed limited experiments to test the effects of gap spacing (of which they found none) and solids volume fraction. Uejima also found that ER fiuid rheology, at least for the shear stress, was fairly well modeled as a Bingham plastic with the shear stress $\tau$ given by

$$
\tau=\tau_{B}+\eta_{B} \dot{\gamma}
$$

where $\tau_{B}$ and $\eta_{B}$ are the so-called Bingham or dynamic yield stress and plastic viscosity, and $\dot{\gamma}$ is the shear rate. Uejima observed that the yield stress scaled with the square of the electric field and the plastic viscosity was approximately constant.

Marshall, Goodwin, and Zukoski ${ }^{9}$ performed a thor- 
ough study of an ER fluid of silica particles in a chlorohydrocarbon suspending fluid. Their experiments covered a large range of field strengths, shear rates, and volume fractions. They found that the effective viscosity of the ER fluid could be correlated with a so-called Mason number, $M a$, a measure of the ratio of viscous forces to electrostatic forces (for example, see Ref. 10). Marshall et al. also noted that the yield stress was linear in the volume fraction range studied. Recent work by Klingenberg ${ }^{11}$ has shown that the yield stress, in fact, has a maximum at around $35-40$ volume percent solids. There have also been several experimental studies on magnetorheological fluids, the magnetic analogs of ER fluids. Work by Mimouni et al. ${ }^{12}$ and Lemaire and Bossis ${ }^{13}$ indicate that these suspension behave quite similarly to ER fluids, and their physics is well described by interacting magnetically induced dipoles. Finally, we point out the excellent review by Gast and Zukoski ${ }^{14}$ that lists several other similar direct and related experimental studies of ER fluids.

There has been some effort to construct micromechanical models of ER fluids with dielectric mismatch as the cause of the electrostatic interactions. Adriani and Gast ${ }^{15}$ developed a perturbation theory to describe an ER fiuid close to its equilibrium balance of Brownian and electrostatic interactions. They calculate the high-frequency elastic modulus and dynamic viscosity of the ER suspension. However, they account for the hydrodynamic and electrostatic interactions from mean-field theories, so their results are not accurate for dense systems. Since the particles are always in close contact when a sufficient electric field is applied, their results are probably only qualitatively correct.

Klingenberg" and Klingenberg, Van Swol, and $\mathrm{Zu}-$ koski $^{16}$ have developed a dynamic simulation of ER fluids. Their method assumes only Stokes drag for the hydrodynamic forces on the particles and simple induced point dipole electrostatic forces, so there are no many-body far-field interactions and no near-field lubrication-like interactions. To prevent particle overlap, a near-field repulsive force is included. Because of the simplicity of their method, they can simulate many particles, and indeed they capture many of the structural features observed in ER suspensions. Their method, however, predicts only the large electric field limit of the effective viscosity, and even then underpredicts experimental results since they do not account for near-field electrostatic interactions. Their simulation also does not provide a good means to develop and test a constitutive model since it contains many approximations. Recently Klingenberg et $a l^{17}$ have included an ad hoc pairwise additive twobody force to account for near-field interactions. We also point out that Whittle ${ }^{18}$ has performed a study with a simulation similar to Klingenberg et al., where he too does not account for the detailed hydrodynamic or electrostatic interactions.

As mentioned earlier, here we develop a dynamic simulation that accurately accounts for both the hydrodynamic and electrostatic interactions. The simulation captures the far- and near-field effects for both types of interactions. The simulation also allows for the prediction of the effective viscosity of the suspension and other rheological properties for any range of electric fields and shear rates. In Sec. II we briefly describe "Stokesian dynamics" which is the basis of our simulation method. Stokesian dynamics simulates the motions of particles due to an imposed bulk shear flow, applied particle forces, and hydrodynamic interactions for zero Reynolds number or inertialess conditions. This technique is accurate over the entire volume fraction range. In addition to the particle motions, the bulk stress of the suspension is computed using Stokesian dynamics from which rheological parameters can be determined.

In order to implement Stokesian dynamics we need the electrostatic interparticle forces and, in Sec. III, we describe a method employing the techniques developed by Bonnecazc and Brady ${ }^{19,20}$ to determine them. This method computes the conservative interparticle force by way of a system electrostatic energy. The system energy includes the effects of both the applied electric field and interparticle interactions. The formulation of the energy using the results of Bonnecaze and Brady allows us to include both the far- and near-field particle interactions. The energy approach developed in this section provides the foundation for computing the strain energy function of an idealized ER fluid and for developing a model for the Bingham or dynamic yield stress in a future paper. ${ }^{21}$ Moreover, this method for computing the manybody electrostatic interparticle force and energy is also valid for any system of particles, not just ER fluids, so it might be useful for other molecular dynamics simulations or other systems of electrically or magnetically interacting particles.

The complete simulation is applied in Sec. IV to an unbounded monolayer of suspended spherical particles, with a particle-to-fluid dielectric constant ratio of 4 , subject to a simultaneous shear flow and orthogonal electric field at an areal fraction of 0.4 for a large range of the Mason number, $M a$. The effective viscosities and first normal stress differences are presented as a function of $M a$. Time traces of the viscosities are also presented along with "snapshots" of the particle configurations; and we discuss the relationship among the microstructure, $M a$, and viscosity. The simulation results are also compared to the experimental data of Marshall et al. ${ }^{9}$ Finally, the effect of periodic boundary conditions is tested and we discuss their effect on the simulation of ER suspensions.

\section{SIMULATION METHOD:}

\section{HYDRODYNAMIC INTERACTIONS AND RHEOLOGY}

\section{A. Fundamentals of Stokesian dynamics}

The Stokesian dynamics method has been described in detail elsewhere, ${ }^{22-24}$ so here we present an outline with special attention to the calculation of the rheology. For $N$ rigid neutrally buoyant particles suspended in an incompressible Newtonian fluid of viscosity $\eta$ and density $\rho$, the motion of the fluid is governed by the Navier-Stokes equations, and the motion of the particles is described by the coupled evolution equation

$$
\mathbf{m} \cdot \frac{d \mathbf{U}}{d t}=\mathbf{F}^{\mathbf{H}}+\mathbf{F}^{\mathbf{P}}
$$

which simply states the following: mass $\times$ acceleration equals the sum of the forces. In Eq. (2) $\mathbf{m}$ is a generalized 
mass/moment of inertia matrix of dimension $6 N \times 6 N$, $U$ is the particle translational/rotational velocity vector of dimension $6 N$, and the $6 N$ force/torque vector $F$ is represented by (i) the hydrodynamic forces $\mathbf{F}^{\mathbf{H}}$ exerted on the particles due to their motion relative to the fluid; and (ii) the deterministic nonhydrodynamic forces, $\mathbf{F}^{\mathbf{P}}$, which for an ER fluid are the interparticle electrostatic forces. A stochastic or Brownian force may also be included in (ii) but is not important here since most ER suspensions are dominated by hydrodynamic and electrostatic forces.

When the motion of the particles and the fluid is on a scale such that viscous forces dominate inertial forces, the fluid dynamics is governed by Stokes equations. In this case, for a small particle Reynolds number $\left(\operatorname{Re}=\rho \gamma a^{2} / \eta \ll 1\right)$, the hydrodynamic force exerted on a particle in a suspension undergoing a bulk shear flow is given by ${ }^{25}$

$$
\mathbf{F}^{\mathbf{H}}=-\mathbf{R}_{F U} \cdot\left(\mathbf{U}-\mathbf{U}^{\infty}\right)+\mathbf{R}_{F E}: \mathbf{E}^{\infty} .
$$

Here, $\mathbf{U}^{\infty}$ is the imposed flow at infinity evaluated at the particle center, $\mathbf{E}^{\infty}$ is the symmetric (and traceless, by virtue of continuity) part of the velocity gradient tensor. The tensors $\mathbf{R}_{F U}$ and $\mathbf{R}_{F E}$ are the hydrodynamic resistance matrices that relate the hydrodynamic force/torque on the particles to their motion relative to the fluid and to the imposed shear flow, respectively. There is a linear relationship between the hydrodynamic force and the velocity and rate of strain because Stokes equations and the boundary conditions are linear. The resistance matrices are purely geometric quantities completely determined by the instantaneous particle configurations. The resistance matrices here and following are carefully formulated to include the long-ranged far-field interactions and the near-field interactions. The long and detailed expressions for these resistance matrices are given in Refs. 23 and 24. The near-field effects for the hydrodynamics are the lubrication forces that exist between two particles in relative motion. Two nearby particles moving toward each other, for example, must squeeze out the thin lubricating film of fluid in the small gap between them. This creates large pressures that generate singular hydrodynamic forces on the particles. These forces are $O\left(\xi^{-1}\right)$ and $O(\ln \xi)$, where $\xi$ is the nondimensionalized gap width between particles. Near-field lubrication is important for preventing particle overlap in the dynamic simulation as well as capturing the effects of percolating chains of particles which we expect to be common in ER fluids.

Since inertial forces are negligible in the limit of small $\mathrm{Re}$, we may neglect the left-hand side (lhs) of Eq. (2) and, in combination with Eq. (3), the equation of motion becomes

$$
\frac{d \mathbf{x}}{d t}=\mathbf{U}=\mathbf{U}^{\infty}+\mathbf{R}_{F U}^{-1} \cdot\left(\mathbf{F}^{\mathbf{P}}+\mathbf{R}_{F E}: \mathbf{E}^{\infty}\right) \text {. }
$$

Nondimensionalizing Eq. (4) and integrating it over a timestep $\Delta t$ produces the evolution equation for the change in particle configuration $\mathbf{x}$,

$$
\Delta \mathbf{x}=\left[\mathbf{U}^{\infty}+\mathbf{R}_{F U}^{-1} \cdot\left(M a^{-1} \mathbf{F}^{\mathbf{P}}+\mathbf{R}_{F E}: \mathbf{E}^{\infty}\right)\right] \Delta t .
$$

Here, $\mathrm{x}$ has been nondimensionalized by the characteristic particle size $a$; the time by the inverse shear rate $\dot{\gamma}^{-1}$; the hydrodynamic forces by $6 \pi \eta a^{2} \dot{\gamma}$; the interparticle electrostatic force by $12 \pi \epsilon a^{2}(\beta E)^{2}$. The so-called Mason number,
$M a=\eta \dot{\gamma} / 2 \epsilon(B E)^{2}$, measures the relative importance of the viscous shear forces to the electrostatic forces. Here $\epsilon$ is the dielectric constant of the fluid, $\epsilon_{p}$ is the dielectric constant of the particle, $\beta=\left(\epsilon_{p}-\epsilon\right) /\left(\epsilon_{p}+2 \epsilon\right)$, and $E$ is the magnitude of the imposed electric field (not to be confused with the bulk rate of strain $\mathbf{E}^{\infty}$ ). Equation (5) clearly depends upon $M a$, but it is also a function of the volume fraction of particles $\phi$ and the electrostatic interparticle forces which are characterized by the dielectric mismatch $\beta$. Note that up to this point no restrictions have been made on particle shape or size.

The suspension bulk stress $\langle\Sigma\rangle$ provides information about the rheology and can also be computed with Stokesian dynamics. The bulk stress is defined as the ensemble or equivalent volume average stress of a statistically homogeneous suspension of particles. For $N$ particles in a volume $V$, Batchelor ${ }^{4.26}$ has shown that

$$
\langle\Sigma\rangle=\mathrm{IT}+2 \eta \mathbf{E}^{\infty}+\frac{N}{V}\left(\left\langle\mathbf{S}^{\mathbf{H}}\right\rangle+\left\langle\mathbf{S}^{\mathbf{P}}\right\rangle-\left\langle\mathbf{x} \mathbf{F}^{\mathbf{P}}\right\rangle\right) .
$$

Here $N / V=n$ is the number density of particles and the terms in angular brackets represent an ensemble average. IT stands for an isotropic term of no interest for incompressible suspensions, and the second term is the fluids' contribution to the stress. The presence of the particles makes the last three contributions to the bulk stress. The hydrodynamic and particle stresslets, $\mathbf{S}^{\mathbf{H}}$ and $\mathbf{S}^{\mathbf{P}}$, are the symmetric and traceless first moments of the force distribution on a particle due to the shear flow and the interparticle forces, respectively. The stresslet is defined by the integral over the particle surface,

$$
\mathbf{S}=\int_{\not \Omega}\left[(\mathbf{x} \sigma+\sigma \mathbf{x}) \cdot \mathbf{n}-\frac{2}{3} \mathbf{I}(\mathbf{x} \cdot \sigma \cdot \mathbf{n})-\eta(\mathbf{u n}+\mathbf{n u})\right] d A,
$$

where $\mathbf{x}$ here is measured relative to some reference point in the particle, $\mathbf{n}$ is the outward facing normal, and $\sigma$ and $\mathbf{u}$ are the stress and velocity on the boundary of the particle for either a shear flow or interparticle forces depending upon whether we are computing $\mathbf{S}^{\mathbf{H}}$ or $\mathbf{S}^{\mathrm{P}}$. The $\partial \Omega$ is the area of the particle surface. Again, due to the linearity of Stokes equations, the stresslets can be written in terms of resistance tensors as

$$
\begin{aligned}
& \left\langle\mathbf{S}^{\mathrm{H}}\right\rangle=-\left\langle\mathbf{R}_{S U} \cdot \mathbf{R}_{F U}^{-1} \cdot \mathbf{R}_{F E}-\mathbf{R}_{S E}\right\rangle: \mathbf{E}^{\infty}, \\
& \left\langle\mathbf{S}^{\mathbf{P}}\right\rangle=-\left\langle\mathbf{R}_{S U} \cdot \mathbf{R}_{F U}^{-1} \cdot \mathbf{F}^{\mathbf{P}}\right\rangle,
\end{aligned}
$$

where $\mathbf{R}_{S U}$ and $\mathbf{R}_{S E}$ are the configuration dependent resistance matrices that relate the particle stresslets to particle velocities and rate of strain. ${ }^{22}$ Generally speaking, the stresslets are the added mechanical or contact stress due to the resistance of the rigid particles to the local deformation of the fluid.

The $-n\left\langle\mathbf{x F}^{\mathrm{P}}\right\rangle$ derived by Batchelor ${ }^{26}$ from virtual strain arguments is the thermodynamic or elastic stress associated with the suspension. The ensemble average $\left\langle\mathbf{x F}^{\mathbf{P}}\right\rangle$ for a statistically homogeneous suspension is given by

$$
\left\langle\mathbf{x} \mathbf{F}^{\mathbf{P}}\right\rangle=\frac{1}{2 N} \sum_{\alpha}^{N} \sum_{\beta}^{N}\left(\mathbf{x}_{\alpha}-\mathbf{x}_{\beta}\right) \mathbf{r}^{\alpha \beta},
$$

where 


$$
\mathbf{F}^{\alpha}=\sum_{\beta}^{N} \mathrm{f}^{\alpha \beta}
$$

Note from the symmetry of the pairwise electrostatic forces $f^{\alpha \beta}=-f^{\beta r z}$. When modeling the suspension rheology by accounting for only a finite number of particle interactions, the individual sums in Eq. (9) are uniformly convergent, and using Eq. (10), gives the thermodynamic stress simply as

$$
\left\langle\mathbf{x} \mathbf{F}^{\mathbf{P}}\right\rangle=\frac{1}{N} \sum_{\alpha}^{N} \mathbf{x}_{\alpha} \mathbf{F}^{\alpha} .
$$

The thermodynamic stress can be interpreted as a spring-like elastic restoring force. Recall that the particles prefer to be aligned with the electric field, so any deformation of that state will produce interparticle forces acting to realign the particle chains. These forces manifest themselves macroscopically as a bulk stress.

Equations (5) and (6) are the core of the dynamic simulation. Given an initial configuration, Eq. (5) is integrated in time to follow the dynamic evolution of the suspension $\mathrm{mi}$ crostructure, and Eq. (6) is evaluated to determine the rheological behavior of the suspension. Clearly from Eqs. (5) and (6), the entire evolution and rheological properties are determined by the hydrodynamic interactions included in the resistance matrices and the electrostatic forces. Stokesian dynamics accurately determines the hydrodynamic resistance matrices so all that is left is the computation of the electrostatic interparticle forces. Before proceeding to that presentation in Sec. III, we shall describe in more detail the contributions to the bulk stress.

\section{B. Contributions to the bulk stress}

In order to better interpret the effective viscosities predicted by the simulation, it would help to understand the three-particle contributions to the bulk stress. The hydrodynamic stress $\mathbf{S}^{\mathbf{H}}$ is the added mechanical stress or dissipation due to the presence of the particles in the flow field. Einstein's $O(\phi)$ correction for viscosity comes from $\mathbf{S}^{\mathbf{H}}$. The hydrodynamic stress is quite sensitive to the suspension microstructure and, for example, can be very large for rodlike chains. In a shear flow the hydrodynamic stresslet of a particle scales roughly as the cube of its largest dimension. Therefore, an ER suspension of particle chains has a higher viscosity compared to the same well-dispersed suspension partially by virtue of the increased hydrodynamic stress. Moreover, relative motion between two nearby particles creates large stresses due to the singular lubrication interaction discussed earlier.

The particle stresslet $\mathbf{S}^{\mathbf{P}}$ is rather different in that its contribution to the viscosity is usually negative for an ER fluid. Consider, for example, two particles in a shear flow and orthogonal electric field as depicted in Fig. 2(a). Now for a spherical particle $\alpha$ its total stresslet is given by the Faxén law ${ }^{23}$

$$
\mathbf{S}=\frac{20}{3} \pi \eta a^{3}\left(1+\frac{a^{2}}{10} \nabla^{2}\right) \mathbf{e}\left(\mathbf{x}_{\alpha}\right),
$$

where $\mathbf{e}\left(\mathbf{x}_{\alpha}\right)$ is the local rate of strain at the center of the sphere that would exist in its absence. Because Stokes equa-
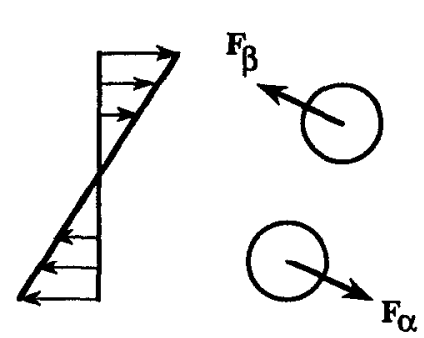

Velocity Gradient on $\beta$ due to $F_{\alpha}$

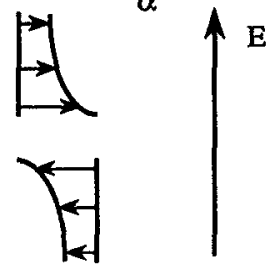

Velocity Gradient on $\alpha$ due to $F_{\beta}$

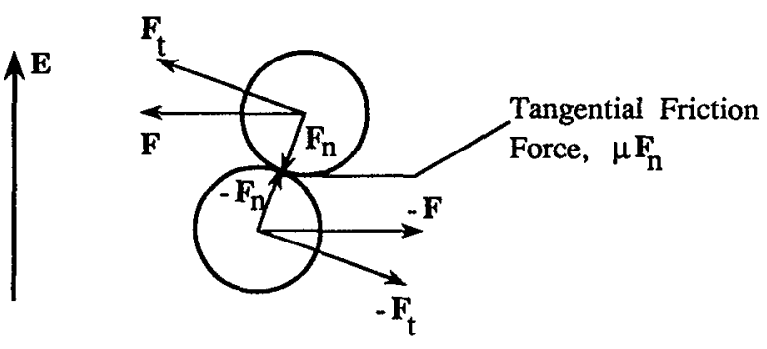

FIG. 2. The effects of stress in a suspension due to electrostatic interparticle forces. While the $-\left\langle\mathbf{x F}^{\mathbf{P}}\right\rangle$ due to chain deformation increases the stress of the suspension, the velocity gradients due to the (a) interparticle forces act counter to the bulk shear flow and reduce the total stress in the suspension. This reduction is not atypical and is also seen, for example, for contact friction forces as depicted in (b).

tions are linear, we can break the computation of $\mathbf{S}$ into that due to the bulk shear flow $\mathbf{S}^{\mathbf{H}}$ and that due to the interparticle forces $\mathbf{S}^{\mathbf{P}}$. If for the hydrodynamic stress we assume as a first approximation that the local strain rate is the bulk strain rate and, since the effective viscosity is the stress divided by the strain rate, the contribution of $\mathbf{S}^{\mathbf{H}}$ to the viscosity is always positive. To determine the contribution of $\mathbf{S}^{\mathbf{P}}$, we must consider the velocity disturbance on one particle due to the force on another. Because of the relative positions of the particles in Fig. 2(a), the resultant electrostatic forces on particle $\alpha$ creates a velocity gradient opposite to that of the bulk flow on particle $\beta$ and likewise for $\beta$ on $\alpha$. Therefore, for this example microstructure, the appropriate component of $\mathbf{S}^{\mathbf{P}}$ must be opposite to its counterpart of $\mathbf{S}^{\mathbf{H}}$ from $\mathbf{E q}$. (12) and, hence, contributes a negative viscosity. The particular geometry shown in Fig. 2(a) is what one would typically expect in an ER fluid, where the shear flow has distorted the alignment of a pair or chain of particles. Occasionally, the suspension might have a microstructure that has the mirror image of that in Fig. 2(a), where $\mathbf{S}^{\mathbf{P}}$ would contribute positively to the viscosity. This, however, occurs infrequently.

The $-\left\langle x F^{P}\right\rangle$ piece for the system in Fig. 2(a) contributes positively to the suspension viscosity, since it resists motion in the direction of the bulk shear flow. The effect of $\mathbf{S}^{\mathbf{P}}$ then appears to be a reduction of the effect of the spring-like restoring force. This is perhaps clearer if we consider the 
situation in Fig. 2(b). The two particles are in contact with equal but opposite forces. The normal forces make no contribution to the stress of the system if there is no deformation of the particles. This is because the stress is concentrated at the point of contact, so the normal force contributions to the particle and thermodynamic stress are

$$
\begin{aligned}
& \left\langle\mathbf{S}^{\mathbf{P}}\right\rangle=-\frac{1}{2}\left(\mathbf{x}_{\alpha}-\mathbf{x}_{\beta}\right) \mathbf{F}_{n}, \\
& -\left\langle\mathbf{x F}^{\mathbf{P}}\right\rangle=\frac{1}{2}\left(\mathbf{x}_{\alpha}-\mathbf{x}_{\beta}\right) \mathbf{F}_{n},
\end{aligned}
$$

and exactly cancel. [Considerations of the form of $\mathbf{R}_{S U} \cdot \mathbf{R}_{F U}^{-1} \cdot \mathbf{F}^{\mathrm{P}}$ near contact will produce Eq. (13).]

The tangential forces do contribute to the particle stress, but depend upon the friction at contact. If we assume, for example, the frictional force to be the product of a friction coefficient $\mu$ and the normal force, then the added stress is,

$$
\langle\Sigma\rangle=\frac{1}{2}\left(\mathbf{x}_{\alpha}-\mathbf{x}_{\beta}\right) \mathbf{F}_{t}-\frac{1}{2}\left(\mathbf{x}_{\alpha}-\mathbf{x}_{\beta}\right) \mu \mathbf{F}_{n}
$$

The first term is the springlike restoring force, and the second term is essentially the $-\left\langle\mathbf{R}_{S U} \cdot \mathbf{R}_{F U}^{-1} \cdot \mathbf{F}^{\mathbf{P}}\right\rangle$ contribution, which is opposite in sign to the first term. In our case, instead of a solid sliding friction coefficient $\mu$, the hydrodynamic lubrication friction of the thin film of fluid between the particles reduces the stress transfer from one sphere to another. From this example this reduction in the stress is clearly not peculiar to just the hydrodynamic interactions of Stokes flow, but is generic.

The important conclusion here is that the reduction of the stress will occur due to any balancing force preventing the spheres from overlapping or running through each other. If some near-field repulsive forces prevented particle overlap, then their contribution to the $-\left\langle\mathbf{x F}^{\mathbf{P}}\right\rangle$ piece would reduce the stress just like the hydrodynamic $\mathbf{S}^{\mathbf{P}}$. Similarly, if particles were allowed to touch and deformed under contact to arrest their motion, then this added deformation stress would play the role of $\mathbf{S}^{\mathbf{P}}$. Using the the strong lubrication forces as the means to prevent particle overlap is particularly convenient from the point of view of implementation since they already exist as part of the suspensions hydrodynamics and do not require the hypothesis of another interparticle force, with its attendent addition of other dimensionless parameters.

We also note here that the contribution of the thermodynamic stress is antisymmetric. As pointed out by Batchelor, ${ }^{4}$ the existence of body couples on particles in a suspension create antisymmetric stresses. The individual spherical particles of an ER suspension have no body couple since the dipole moment and the electric field are aligned. The chain of particles, however, does have a net torque trying to realign it with the electric field. Considering each chain as a single body, there are then indeed body couples and, thus, there are antisymmetric stresses. Close examination of $-n\left\langle\mathbf{x} \mathbf{F}^{\mathbf{P}}\right\rangle$ in the forthcoming simulations does show the stresses to be antisymmetric. This is, however, not of much importance for an ER device where the simple shear flow is always perpendicular to the applied electric field.

\section{SIMULATION METHOD: ELECTROSTATIC INTERPARTICLE FORCES}

The exact electrostatic interparticle forces in an ER suspension can be computed from the solution of the manybody potential problem and the integration of Maxwell stresses over each particle. This is practically impossible, however, for anything more than two spherical particles. There is an alternative method that avoids the need for detailed knowledge of the local potential field that is quite simple and elegant-using the electrostatic energy of the system. Since the electrostatic interparticle force is conservative, the force on any particle is the negative of the gradient of the electrostatic energy with respect to the particle's position, and this energy can be written in terms of a geometric system capacitance matrix.

Recall that the energy of a system of charged particles in the absence of an electric field is one-half the sum of the product of the particle charges and their local potentials. ${ }^{27.28}$ For charge-free particles in an electric field, the electrostatic energy density is analogously given by ${ }^{28}$

$$
\mathscr{U}=\frac{1}{2 V} \sum_{\alpha} \mathbf{S}_{E}^{\alpha} \cdot \mathbf{E}=\frac{1}{2 V} \mathbf{S}_{E} \cdot \mathscr{E} .
$$

Here $\mathscr{Z}$ is the energy per unit volume for a system of $N$ particles in a volume $V$. The induced dipole on particle $\alpha$ is $\mathbf{S}_{E}^{\alpha}$ and $\mathbf{E}$ is the macroscopic applied electric field. The slightly more compact form on the rhs of Eq. (16) has $\mathbf{S}_{E}$ as the vector containing the $3 N$ components of all the particle dipoles and $\mathscr{E}$ as the complementary $3 N$ electric field vector.

Since Laplace's equation and the boundary conditions are linear in the electric field, there exists the relationship

$$
\left(\begin{array}{c}
\mathbf{q} \\
\mathbf{S}_{E}
\end{array}\right)=\left(\begin{array}{ll}
\mathbf{C}_{q \Phi} & \mathbf{C}_{q E} \\
\mathbf{C}_{S \Phi} & \mathbf{C}_{S E}
\end{array}\right) \cdot\left(\begin{array}{c}
\Phi \\
\mathscr{E}
\end{array}\right)=\mathbf{C} \cdot\left(\begin{array}{c}
\Phi \\
\mathscr{E}
\end{array}\right),
$$

where $q$ and $\Phi$ are the $N$-dimensional vectors containing the particle charges and potentials, and $\mathbf{C}$ is the so-called grand capacitance matrix and its submatrices, $\mathbf{C}_{q \phi}$ for example, relate the particle charges to their potentials, etc. For charge-free particles,

$$
\mathbf{S}_{E}=\widehat{\mathbf{C}}(\mathbf{x}) \cdot \mathscr{E},
$$

where, from solving Eq. (17) for $q=0$,

$$
\widehat{\mathbf{C}}(\mathbf{x})=\mathbf{C}_{S \phi} \cdot\left(\mathbf{C}_{q \phi}\right)^{-1} \cdot \mathbf{C}_{q E}-\mathbf{C}_{S E} \text {. }
$$

Here $\widehat{\mathbf{C}}(\mathbf{x})$ is the dipole electric field capacitance matrix for charge-free particles and the $x$ reminds us that it (and all the capacitance matrices) are functions of the instantaneous particle positions like the hydrodynamic resistance matrices. The capacitance matrices are also a function of the particleto-fluid dielectric constant ratio.

The electrostatic energy density is now

$$
\mathscr{U}=\frac{1}{2 V} \mathscr{E} \cdot \widehat{\mathbf{C}}(\mathbf{x}) \cdot \mathscr{E}
$$

and then, from classical physics, the force on particle $\alpha$ is

$$
\mathbf{F}^{\alpha}=-\frac{\partial \mathscr{U}(\mathbf{x})}{\partial \mathbf{x}_{\alpha}}=-\frac{1}{2} \mathscr{E} \cdot \frac{\partial \widehat{\mathbf{C}}(\mathbf{x})}{\partial \mathbf{x}_{\alpha}} \cdot \mathscr{E},
$$

and, similarly, the torque on the particle is 


$$
\mathbf{T}^{\alpha}=-\frac{\partial \mathscr{U}(\mathbf{x})}{\partial \psi_{\alpha}}=-\frac{1}{2} \mathscr{E} \cdot \frac{\partial \hat{\mathbf{C}}(\mathbf{x})}{\partial \psi_{\alpha}} \cdot \mathscr{E},
$$

where $\mathbf{x}_{\alpha}$ and $\psi_{\alpha}$ are the particle's position and Euler angle vectors. Here we see clearly that there is no torque on a spherical particle since its Euler angle is irrelevant to its dipole strength and the system electrostatic energy. Also note that both the energy and the interparticle force scale with the square of the electric field. The pairwise particle forces $f^{\alpha \beta}$ are determined from the energy as well as

$$
\mathbf{f}^{\alpha \beta}=-\frac{\partial \mathscr{U}(\mathbf{x})}{\partial\left(\mathbf{x}_{\alpha}-\mathbf{x}_{\beta}\right)}=-\frac{1}{2} \mathscr{E} \cdot \frac{\partial \widehat{\mathbf{C}}(\mathbf{x})}{\partial\left(\mathbf{x}_{\alpha}-\mathbf{x}_{\beta}\right)} \cdot \mathscr{C},
$$

and are used for the computation of the thermodynamic stress in Eq. (9). A similar expression can be constructed for the pairwise particle torques.

The grand capacitance matrix and all its submatrices are formed to include both the long-ranged many-body interactions and the lubrication-like near-field interactions. We do so by the method of Bonnecaze and Brady ${ }^{19}$ which approximates the grand capacitance matrix by

$$
\mathbf{C} \approx \mathbf{M}^{-1}+\mathbf{C}_{2 b} \cdots \mathbf{C}_{2 b}^{\infty} .
$$

The potential matrix $\mathbf{M}$ in its exact form is the inverse of the capacitance matrix. Here, we form an approximation to the potential matrix from a moment expansion about each particle derived from the integral representation of Laplace's equation. The moment expansion is done through the dipole level and the $\mathbf{M}$ can be written as

$$
\mathbf{M}=\left(\begin{array}{ll}
\mathbf{M}_{\phi q} & \mathbf{M}_{\phi S} \\
\mathbf{M}_{E q} & \mathbf{M}_{E S}
\end{array}\right),
$$

where $\mathbf{M}_{\phi q}$ relates the particle potentials to their charges, etc. If additional moments are included in the approximation, they can also be solved for in terms of $q$ and $\mathbf{S}_{E}$ and thus written in the form of Eq. (25). The detailed expressions for the potential and capacitance matrices are in Ref. 19.

The accuracy of the potential matrix and, hence, the accuracy of its inverse depends upon the number of moments retained in its formulation. The inversion of the potential matrix captures the many-body far-field particle interactions since the inversion is equivalent to a many-body method of reflections solution. The lubrication-like near-field interactions for particles near contact are captured by direct addition of the exact two-body capacitance matrix, $\mathbf{C}_{2 b}$, less the far-field two-body capacitance matrix, $\mathbf{C}_{2 b}^{\infty}$, already included in the potential invert. This method has already proven very effective for computing the effective conductivity of a suspension, which is proportional to the average suspension dipole. ${ }^{20}$

Since the capacitance matrix includes both the far- and near-field interactions, so do the interparticle forces. The near-field interactions are, in fact, quite strong, and for perfect conductors $\left(\epsilon_{p} / \epsilon=\infty\right)$, the interparticle force scales as $O\left(\xi^{-1}\right)$ for nearby particles as shown by Jeffrey. ${ }^{29}$ This singularity is captured by the energy formulation. Recall ${ }^{19}$ that the dipoles for two particles near contact in an electric field are $O(\ln \xi)$. Thus, from Eq. (16), the energy is also $O(\ln \xi)$ and the derivative of the energy with respect to sepa- ration or force is $O\left(\xi^{-1}\right)$. The near-field interactions are smaller for the more modest dielectric constant ratios, but there is still an enhancement in the interparticle force over the many-body far-field effects. The relative importance of the near-field interactions to the far-field interactions decreases as the particle-to-fluid dielectric constant ratio approaches unity.

It is interesting to know the form of the resulting force if no near-field interactions are included in the capacitance matrix. Using just the potential matrix of Eq. (25), we find that

$$
\mathbf{S}_{E}=\mathbf{M}_{E S}^{-1} \cdot \mathscr{C}
$$

and, thus,

$$
\mathbf{F}^{\alpha}=-\frac{1}{2} \mathscr{E} \cdot \frac{\partial \mathbf{M}_{E S}^{-1}}{\partial \mathbf{x}_{\alpha}} \cdot \mathscr{E} .
$$

A similar expression can be written for the torque. Because we can determine $\mathbf{M}_{E S}$ more readily than its inverse, we rewrite Eq. (27) to remove the derivative of the invert using a matrix identity to yield

$$
\mathbf{F}^{\alpha}=\frac{1}{2} \mathscr{E} \cdot \mathbf{M}_{E S}^{-1} \cdot \frac{\partial \mathbf{M}_{E S}}{\partial \mathbf{x}_{\alpha}} \cdot \mathbf{M}_{E S}^{-1} \cdot \mathscr{E} .
$$

Using Eq. (26) and the fact that $\mathbf{M}_{E S}^{-1}$ is symmetric Eq. (28) becomes

$$
\mathbf{F}^{\alpha}=\frac{1}{2} \mathbf{S}_{E} \cdot \frac{\partial \mathbf{M}_{E S}}{\partial \mathbf{x}} \cdot \mathbf{S}_{E}
$$

Finally, from the dipole propagator for disturbances to the electric field, ${ }^{19}$ we can expand Eq. (29) with a bit of algebra to yield

$$
\mathbf{F}^{\alpha}=\mathbf{S}_{E}^{\alpha} \cdot \sum_{\substack{\beta=1 \\ \beta \neq a}}^{N} \nabla \nabla \nabla \frac{1}{r} \cdot \mathbf{S}_{E}^{\beta},
$$

where $r=\left|\mathbf{x}_{\alpha}-\mathbf{x}_{\beta}\right|$ is the center to center distance between particles $\alpha$ and $\beta$. Thus, given the particle dipoles computed from the potential invert and the particle positions, we can compute the far-field approximation to the electrostatic interparticle force with Eq. (30). It is important to note here that in Eq. (30), one needs to know the particle dipole $\mathbf{S}_{E}^{\beta}$. This dipole, even when near-field effects are neglected, is not equal to that of an isolated particle, but must be found as a solution of the many-body electrostatic problems via Eq. (18) and the capacitance matrix.

It is also interesting to see how our method for computing the force compares to the methods employed by Klingenberg, Van Swol, and Zukoski ${ }^{16,17}$ and Whittle. ${ }^{18}$ The basis of their method is a multipole expansion of the particle forces. From Jackson ${ }^{27}$ the force on a particle with some charge distribution is approximately given by

$$
\mathbf{F}^{\alpha}=q_{\alpha} \mathbf{E}\left(\mathbf{x}_{\alpha}\right)+\mathbf{S}_{E}^{\alpha} \cdot \nabla \mathbf{E}\left(\mathbf{x}_{\alpha}\right)+\cdots,
$$

where $\mathbf{E}\left(\mathbf{x}_{\alpha}\right)$ is the local electric field evaluated at the center of particle $\alpha$. Equation (31) neglects higher-order moments of the charge distribution and higher-order variations in the local electric field. For $N$ charge-free particles, the local gradient of the electric field is approximately given by ${ }^{19}$ 


$$
\nabla \mathbf{E}\left(\mathbf{x}_{\alpha}\right)=\sum_{\substack{\beta=1 \\ \beta \neq \alpha}}^{N} \nabla \nabla \nabla \frac{1}{r} \cdot \mathbf{S}_{E}^{\beta}+\cdots .
$$

Equation (32) also ignores the higher multipoles of the charge distribution and higher-order variations in the local electric field. Thus combining Eq. (31) with Eq. (32) yields the force on a charge free particle, namely,

$$
\mathbf{F}^{\alpha}=\mathbf{S}_{E}^{\alpha} \cdot \sum_{\substack{\beta=1 \\ \beta \neq \alpha}}^{N} \nabla \nabla \nabla \frac{1}{r} \cdot \mathbf{S}_{E}^{\beta}+\cdots,
$$

which is exactly what we derived in Eq. (30). We see that our method, using only the potential matrix invert, is equivalent to a moment expansion of the force up to the dipole level. The addition of the two-body interactions into the capacitance matrix then includes the effects of the infinite sum of the remaining higher moments and captures the near-field effects that are important for high dielectric constant particles near contact.

Klingenberg et al. ${ }^{16}$ employ Eq. (33) but use the induced dipole of an isolated particle in an electric field, so all the particles have the identical dipole strength. Hence, they neglect many-body far-field interactions as well as the nearfield interactions. Klingenberg et al. ${ }^{17}$ have recently augmented their method to add in an ad hoc manner exact twobody forces to approximate near-field interactions, while still neglecting the many-body effects on the particle dipoles. Whittle ${ }^{18}$ uses a method similar to Klingenberg et al. as the basis to compute the interparticle forces, and he also uses an ad hoc force field to capture the near-field interactions.

Unfortunately, there are limited results available to compare against the many-body electrostatic force computed from the energy formulation via the capacitance matrix. In fact, the only results for modest dielectric constant ratios (10 or less) are the forces for two identical spherical particles in an electric field computed by Klingenberg and presented in Gast and Zukoski. ${ }^{14}$ The force is represented by three functions $f_{\|}, f_{\perp}$, and $f_{\Gamma}$, of center-to-center separation, $r$, in the equation

$$
\begin{aligned}
\mathbf{F}^{\alpha}= & 12 \pi \epsilon_{p}(\beta E)^{2} a^{2}\left(\frac{a}{r}\right)^{4} \\
& \times\left[\left(2 f_{\|} \cos ^{2} \theta-f_{\perp} \sin ^{2} \theta\right) \mathbf{e}_{r}+f_{\Gamma} \sin 2 \theta \mathbf{e}_{\theta}\right] .
\end{aligned}
$$

Here $\theta$ is the angle between the sphere line of centers and the applied electric field, $\mathbf{e}_{r}$ is the unit vector along the sphere line of centers, and $e_{\theta}$ is the azimuthal unit vector. Table I lists the results using the energy formulation with and without the addition of near-field effects with those of Klingenberg for $\epsilon_{p} / \epsilon=2$ and 10 . The energy method results are in excellent agreement with Klingenberg's data with the nearfield interactions. Without the near-field interactions the predicted forces are (within 15\%) for separations as close as 2.2 sphere radii. The error is greater for the higher dielectric constant ratio as the near-field interactions become more important. When the particles are near contact the error is approximately $25 \%$ for $\epsilon_{p} / \epsilon=2$ and $71 \%$ for $\epsilon_{p} / \epsilon=10$. However, our method, with the absence of near-field effects, is still superior to that of point dipoles. For point dipoles the force functions are unity for all particle separations, while
TABLE 1. Comparison of two-sphere force functions. The Klingenberg

\begin{tabular}{|c|c|c|c|c|c|c|c|c|c|}
\hline$r / a$ & $K$ & $\begin{array}{l}\mathrm{NF} \\
f_{\|}\end{array}$ & $\mathrm{FF}$ & $K$ & $\begin{array}{l}\mathrm{NF} \\
f_{1}\end{array}$ & $\mathrm{FF}$ & $K$ & $\begin{array}{l}\mathrm{NF} \\
f_{\Gamma}\end{array}$ & FF \\
\hline \multicolumn{10}{|c|}{$\epsilon_{\rho} / \epsilon=2.0$} \\
\hline 3.0 & 1.09 & 1.09 & 1.06 & 0.97 & 0.97 & 0.97 & 1.01 & 1.01 & 1.01 \\
\hline 2.5 & 1.11 & 1.11 & 1.08 & 0.94 & 0.96 & 0.96 & 1.03 & 1.03 & 1.01 \\
\hline 2.2 & 1.27 & 1.27 & 1.11 & 0.88 & 0.88 & 0.95 & 1.05 & 1.05 & 1.02 \\
\hline 2.0 & 1.50 & 1.50 & 1.14 & 0.82 & 0.82 & 0.93 & 1.08 & 1.08 & 1.04 \\
\hline \multicolumn{10}{|c|}{$\epsilon_{p} / \epsilon=10.0$} \\
\hline 3.0 & 1.18 & 1.18 & 1.14 & 0.92 & 0.92 & 0.92 & 1.04 & 1.04 & 1.03 \\
\hline 2.5 & 1.45 & 1.45 & 1.22 & 0.83 & 0.83 & 0.90 & 1.10 & 1,10 & 1.05 \\
\hline 2.2 & 2.09 & 2.09 & 1.35 & 0.72 & 0.72 & 0.87 & 1.20 & 1.20 & 1.10 \\
\hline 2.0 & 5.20 & 5.17 & 1.51 & 0.62 & 0.63 & 0.83 & 1.46 & 1.44 & 1.15 \\
\hline
\end{tabular}
data from Ref. 14 are under the heading $K$. The NF and FF headings are for the energy formulation of the force with near-field interactions and with only far-field interactions.

Eq. (30) gives the correct result in the far field since the many-body interactions are still included.

As a last remark, we note that determining the particle dipoles for an infinite suspension results in convergence problems of a kind familiar to all long-range interactions. Our method ${ }^{19,20}$ correctly calculates these interactions by properly assessing the effect of far particles and performing the requisite sums. With the electrostatic interparticle forces, we can now implement the dynamic simulation and do so in Sec. IV to study the dynamics and rheology of an ER suspension.

\section{RHEOLOGY AND MICROSTRUCTURE OF A MONOLAYER}

\section{A. Introductory remarks}

We applied the simulation method outlined in the preceding sections to an unbounded monolayer of dielectric spheres with the electric field in the $y$ direction and the shear flow in the $x$ direction as illustrated in Fig. 3. A monolayer is studied for a few reasons. The microstructure is more easily visualized with video animations and instantaneous "snapshots" of the particle configurations. Using the monolayer system also reduces the computation time by almost a factor of 8 compared to a full three-dimensional simulation. It still,

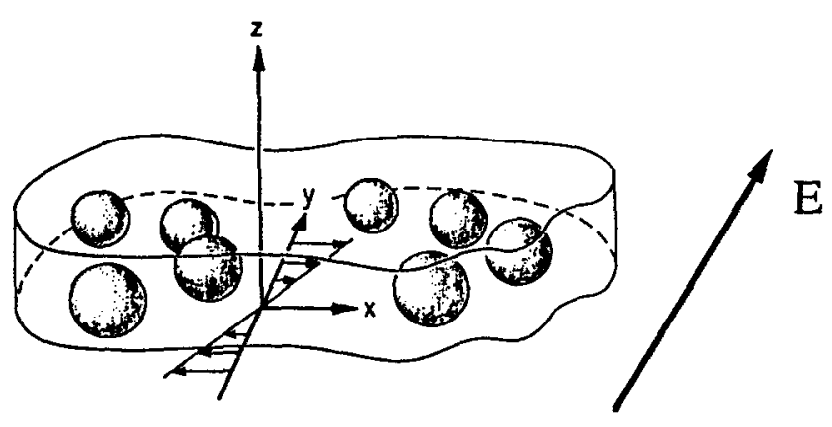

FIG. 3. Illustration of monolayer of spheres used in dynamic simulation. The shear flow in the $x$ direction is orthogonal to the applied electric field in the $y$ direction. To simulate an unbounded suspension, periodic boundary conditions are applied to the $N$ particles in the cell. 
however, retains much of the underlying physics of the problem, as we shall see. Moreover, although the particle centers are confined to a two-dimensional plane (not by applied forces but simply by the symmetry of the hydrodynamic and electrostatic interactions), the interactions are those of three-dimensional spheres. To simulate an unbounded infinite suspension, we apply periodic boundary conditions to a periodic cell containing $N$ particles. Note that in order to sum the three-dimensional interactions, the sheet of particles must also be replicated in the $z$ direction as well. The sheets are placed far enough apart such that the measured effective viscosities are no longer a function of the separation. A distance of 16 particle radii between the sheets was more than sufficient.

In order to compute a viscosity from Eq. (6) for the monolayer, we need the number density of particles, which requires a volume for the $N$ particles in the sheet of a periodic cell. We choose this volume to be the product of the area of the periodic cell and twice the particle radius, or the thickness of the monolayer. The bulk shear is in the $x y$ plane $\left(\mathbf{U}^{\infty}=\dot{\gamma} y \mathbf{e}_{x}\right)$, so the total relative viscosity $\eta^{r}$, the viscosity of the suspension divided by the viscosity of the fluid, is the proportionality between the $y x$ components of the strain and the bulk stress, i.e.,

$$
\eta^{T}=\eta^{H}+\eta^{s^{P}}+\eta^{X F}
$$

where

$$
\begin{aligned}
\eta^{H} & =1+\frac{5}{3} \phi_{A} \frac{1}{N} \sum_{\alpha=1}^{N}\left(\mathbf{S}_{\alpha}^{H}\right)_{y x}, \\
\eta^{S^{P}} & =\frac{5}{3} \phi_{A} \frac{1}{M a N} \sum_{\alpha=1}^{N}\left(\mathbf{S}_{\alpha}^{P}\right)_{y x}, \\
\eta^{X F} & =\frac{5}{3} \phi_{A} \frac{1}{M a 2 N} \sum_{\alpha}^{N} \sum_{\beta}^{N}\left[\left(\mathbf{x}_{\alpha}-\mathbf{x}_{\beta}\right) \mathbf{f}^{\alpha \beta}\right]_{y x} .
\end{aligned}
$$

The areal fraction of the monolayer is $\phi_{A}$. We shall call $\eta^{H}$ the hydrodynamic viscosity, which includes the fluid contribution of unity in Eq. (36a), $\eta^{S^{P}}$ the particle viscosity, and $\eta^{X F}$ as the $\mathbf{x F}^{\mathbf{P}}$ viscosity. Note both the particle and $\mathbf{x F}^{\mathbf{P}}$ viscosity are proportional to $M a^{-1}$, and we shall sometimes refer to their combination as $\eta^{\mathrm{ER}}$, the $\mathrm{ER}$ viscosity. The stresslet in Eq. (36) has been nondimensionalized such that an isolated sphere in the monolayer gives a hydrodynamic contribution to the relative viscosity of $\frac{5}{3} \phi_{A}$, which is the monolayer equivalent of the Einstein viscosity.

\section{B. Sphere ordering in an electric field}

An initial test of the simulation was to see if particle chaining was observed and to compare the predicted chaining time to experimental observations. The spheres were placed randomly within the periodic cell monolayer and allowed to order under the action of an applied electric field. Figure 4 illustrates the progressive development of a microstructure for 25 particles at an areal fraction of $0.4, M a=10^{-3}$. Since there is no shear flow, the choice of $M a$ is arbitrary insofar as the time scales as $\dot{\gamma}^{-1}=M a^{-1} \eta /\left[2 \epsilon(\beta E)^{2}\right]$ in the simulation. The square in Fig. 4 is the outline of the periodic cell. By $t=0.2$, three completc chains have formed and aligned with the electric field. Two of the chains have formed a chain doublet connected by particle $I$. In comparing Figs. $4(\mathrm{a})-4(\mathrm{~b})$, we see the aggregation of nearest neighbors as described in the Introduction. Particles $G-K-P$ have rotated and aligned with the electric field, and particles $W-V-U-Y$ have formed an aligned cluster as well. At $t=2.7$, the three chains have formed a chain triplet with one or two particle bridges. Note that the third chain moves down as well.

At first we might think this is contrary to the arguments made in the Introduction that particles whose line of centers is perpendicular to the electric field repel one another. While this is true for particles directly apart from each other, a particle in one chain also experiences attractive forces from the particles above and below its direct opposite in the other chain. There is, in fact, a net attraction that causes the chains to approach one another. The time for approach, as we see, is much slower than for the initial chain formation because the hydrodynamic resistance is much larger for this long rodlike body while the electrostatic forces are no greater. It is possible, however, that there might exist a "kinetic equilibrium" configuration of chains where the sum of the forces on each particle vanishes as in the case for a periodic array. The final configuration would, in general, depend upon the initial conditions, and it is not clear that such a configuration would be stable to disturbances.

From our simulation we can estimate the chaining time in an ER fluid. The dimensional chaining time is the simulation chaining time multiplied by $M a^{-1} \eta / 2 \epsilon(\beta E)^{2}$. Marshall, Goodwin, and Zukoski ${ }^{16}$ used an ER fluid with $\eta \approx 0.01 \mathrm{~Pa} \mathrm{~s}, \epsilon=8 \times 8.854 \times 10^{-2} \mathrm{farad} / \mathrm{m}$, and an electric field of about $1 \mathrm{kV} / \mathrm{mm}$. For a simulation chaining time of 0.1 , the dimensional chaining time is about $0.03 \mathrm{sec}$. Marshall et al. report that the ER suspension "sets up" in less than $0.1 \mathrm{sec}$ which is consistent with our simulation results. Furthermore, we estimate the dimensional aggregation time of two chains to be approximately $0.8 \mathrm{sec}$ or about 26 times longer than the chaining time, no doubt due to the greater hydrodynamic resistance of the chains.

Experimentally, Conrad, Fisher, and Sprecher ${ }^{30}$ have observed chains of multiple particle thickness. After a short time the chains do not grow in thickness. It is observed that the particles near the electrodes are practically forever attached to the metal surface while there is an electric field. This is probably due to both the strong electrostatic forces with the metallic surface and surface roughness of both the particles and electrodes. The simulation chains will grow no thicker since the periodic boundary conditions preclude the addition of any other chains.

\section{Dynamic simulation results: Effective viscosity}

Several dynamic simulations were performed on an infinite, unbounded monolayer of spherical particles with a particle to the fluid dielectric constant ratio of 4 . The periodic cell was square in shape and there were 25 particles per cell. The simulations were performed with and without the addition of the near-field electrostatic interactions, although near-field hydrodynamic interactions were always included since they are necessary to prevent particle overlap. The sim- 


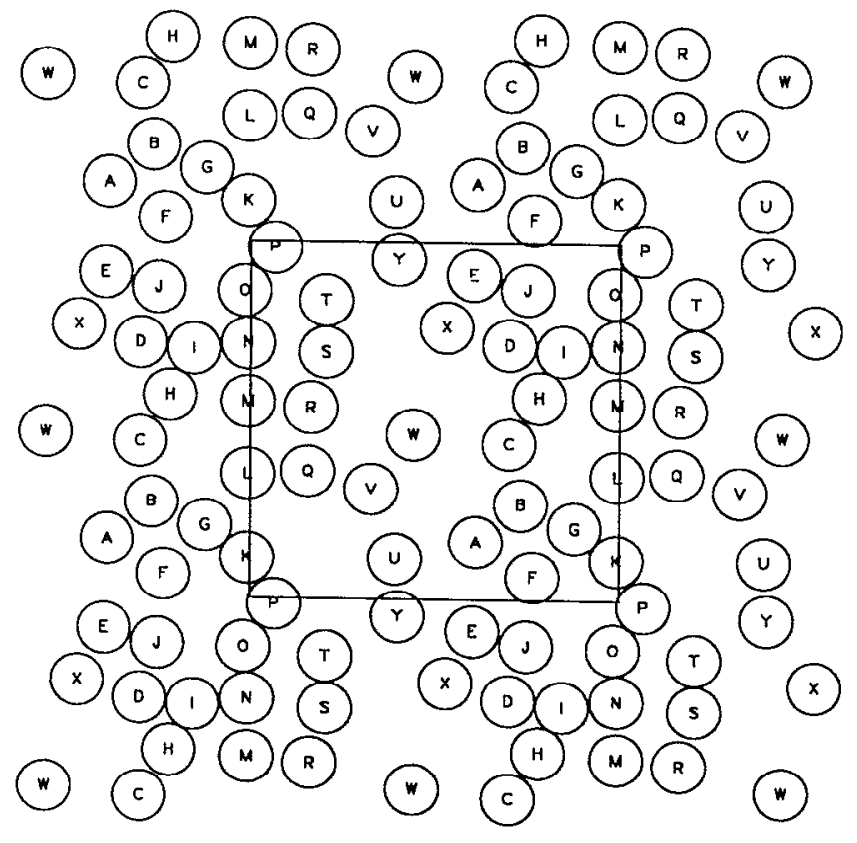

(a)

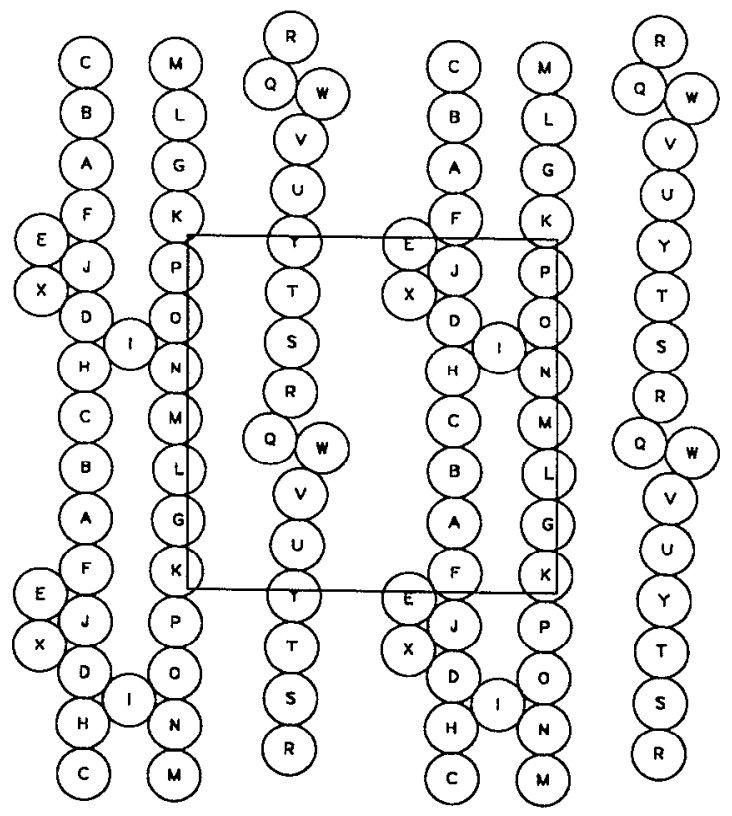

(b)

ulation times $T$ were typically 10.0 (nondimensionalized by the shear rate) but were sometimes less. The CPU time for a simulation increased with decreasing $M a$ since smaller time steps were required to resolve the rapid microstructural rearrangements that occur with strong electric fields. Approximately $5 \mathrm{~h}$ of CPU time on an IBM 520 RISC 6000 machine are needed for a simulation of 25 particles at $M a=10^{-4}$ to a nondimensional time of 10 . Since the completion of this study, coding improvements of the simulation have decreased the execution time by at least a factor of 2 .

In all cases, the reported average of the effective viscosities had reached steady-state values. The summary of the

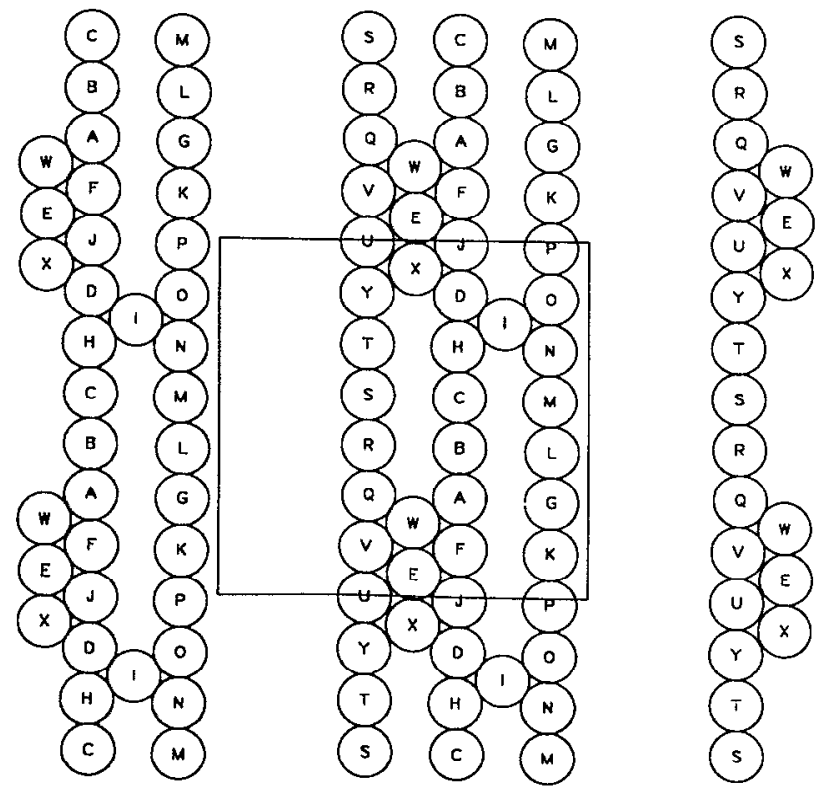

(c)

FIG. 4. Instantaneous "snapshots" of the suspension microstructure for sphere ordering with no shear flow and $M a=10^{-3}$. With no shear-rate time scales as $M a^{-1} \eta /\left[2 \epsilon(\beta E)^{2}\right]$. The outlined square is the periodic ccll. (a) $t=0.0$; (b) $t=0.2$; (c) $t=2.7$. 


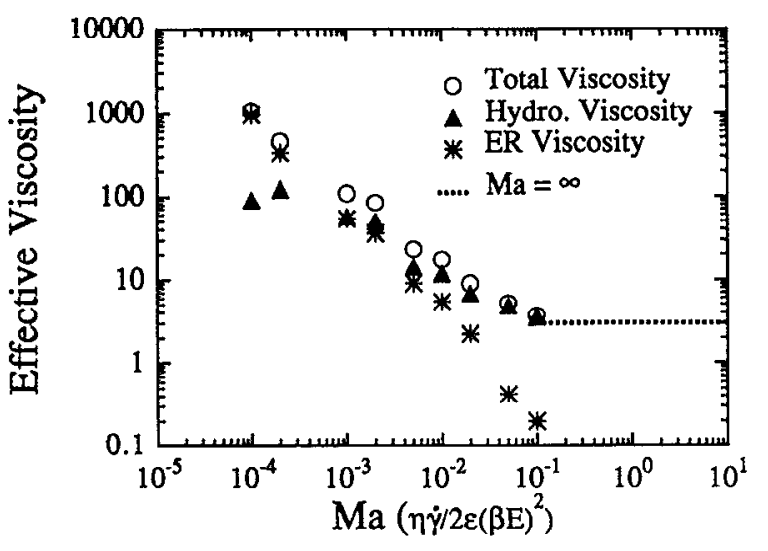

FIG. 5. Total, hydrodynamic, and ER viscosities for monolayer simulations without the addition of near-field electrostatic interactions. The viscosities are relative to the suspending fluid. The dotted line corresponds to the noelectric field viscosity. Table II lists these viscosities including the near-field interactions, and the results are qualitatively similar.

resistance to straining of the particles, increases with decreasing $M a$ up until about $M a=2 \times 10^{-4}$ where it appears to reach a plateau value of about 100 . The ER viscosity steadily increases with decreasing $M a$ over the entire range presented herc. At high $M a$, when the viscous forces dominate, the viscosity is almost entirely the hydrodynamic contribution. Note the total viscosity is approximately equal to the $M a=\infty$ limit (pure shear, no electrostatics) at about $M a=10^{-1}$. As the $M a$ decreases, the ER viscosity contri-

TABLE II. Dynamic simulation effective viscosities. All viscosities reported are relative to the suspending fluid viscosity $\eta$. The time $T$ is the time of the simulation and is nondimensionalized with the shear rate $\dot{\gamma}$. The time steps for each simulation varied from $10^{-3}$ for $M a=\infty$ to $2 \times 10^{-3}$ for $M a=1 \times 10^{-4}$. The smaller time steps are needed to resolve the rapid reconfigurations that occur on a much shorter time scale.

\begin{tabular}{|c|c|c|c|c|c|c|}
\hline$M a$ & $\left\langle\eta^{\prime}\right\rangle$ & $\left\langle\eta^{\prime \prime}\right\rangle$ & $\left\langle\eta^{s^{r}}\right\rangle$ & $\left\langle\eta^{X F}\right\rangle$ & $\left\langle\eta^{\mathrm{ER}}\right\rangle$ & $T$ \\
\hline \multicolumn{7}{|c|}{$N=25$, no near-field electrostatic interactions } \\
\hline$\infty$ & 2.85 & 2.85 & 0.0 & 0.0 & 0.0 & 10.1 \\
\hline $10^{-1}$ & 3.68 & 3.48 & -0.10 & 0.30 & 0.20 & 10.1 \\
\hline $5 \times 10^{-2}$ & 5.20 & 4.78 & -0.24 & 0.66 & 0.42 & 6.0 \\
\hline $2 \times 10^{-2}$ & 9.03 & 6.78 & -2.16 & 4.41 & 2.25 & 10.1 \\
\hline $10^{2}$ & 17.33 & 11.84 & -4.40 & 9.89 & 5.49 & 10.1 \\
\hline $5 \times 10^{-3}$ & 23.20 & 14.03 & -8.04 & 17.21 & 9.17 & 3.0 \\
\hline $2 \times 10^{-3}$ & 85.13 & 49.08 & -12.09 & 48.14 & 36.05 & 10.1 \\
\hline $10^{-3}$ & 109.6 & 55.79 & -23.67 & 77.43 & 53.76 & 10.1 \\
\hline $2 \times 10^{-4}$ & 452.7 & 120.8 & -14.61 & 346.5 & 331.9 & 5.1 \\
\hline $10^{-4}$ & 1055 & 90.26 & -310.8 & 1275 & 964.2 & 10.1 \\
\hline \multicolumn{7}{|c|}{$N=25$, near-field electrostatic interactions } \\
\hline$\infty$ & 2.85 & 2.85 & 0.0 & 0.0 & 0.0 & 10.1 \\
\hline $10^{-1}$ & 3.77 & 3.43 & -0.19 & 0.53 & 0.34 & 10.1 \\
\hline $10^{2}$ & 22.10 & 15.51 & -12.89 & 19.48 & 6.59 & 10.1 \\
\hline $10^{3}$ & 120.3 & 61.32 & -29.74 & 88.68 & 58.94 & 10.1 \\
\hline $2 \times 10^{4}$ & 544.4 & 120.0 & -344.6 & 769.0 & 424.4 & 4.1 \\
\hline $10^{-4}$ & 1248 & 102.7 & -1593 & 2738 & 1145 & 8.85 \\
\hline \multicolumn{7}{|c|}{$N=49$, no near-field electrostatic interactions } \\
\hline $10^{-2}$ & 13.15 & 8.42 & -2.31 & 7.04 & 4.73 & 8.0 \\
\hline $10^{-3}$ & 114.0 & 52.06 & -26.70 & 88.64 & 61.94 & 2.0 \\
\hline \multicolumn{7}{|c|}{$N=50$ (rectangular box), no near-field electrostatic interactions } \\
\hline $10^{2}$ & 16.82 & 14.70 & -1.84 & 3.96 & 2.12 & 22.0 \\
\hline $10^{-3}$ & 93.22 & 56.04 & -12.00 & 49.18 & 37.18 & 10.0 \\
\hline $10^{-4}$ & 444.1 & 96.74 & -123.7 & 471.1 & 347.4 & 2.6 \\
\hline
\end{tabular}

TABLE III. 95\% confidence limits for viscosities in Table II.

\begin{tabular}{|c|c|c|c|c|}
\hline$M a$ & $\left\langle\eta^{T}\right\rangle$ & $\left\langle\eta^{\prime \prime}\right\rangle$ & $\left\langle\eta^{s^{n}}\right\rangle$ & $\left\langle\eta^{x+}\right\rangle$ \\
\hline \multicolumn{5}{|c|}{$N=25$, no near-field electrostatic interactions } \\
\hline$\infty$ & 0.10 & 0.10 & 0.0 & 0.0 \\
\hline $10^{-1}$ & 0.11 & 0.11 & 0.05 & 0.10 \\
\hline $5 \times 10^{-2}$ & 0.11 & 0.13 & 0.13 & 0.09 \\
\hline $2 \times 10^{-2}$ & 0.22 & 0.18 & 0.08 & 0.11 \\
\hline $10^{-2}$ & 0.68 & 0.59 & 0.19 & 0.18 \\
\hline $5 \times 10^{-3}$ & 1.01 & 1.14 & 0.93 & 0.43 \\
\hline $2 \times 10^{-3}$ & 5.48 & 4.85 & 1.54 & 0.84 \\
\hline $10^{-3}$ & 11.76 & 12.35 & 3.48 & 2.20 \\
\hline $2 \times 10^{-4}$ & 49.1 & 37.9 & 23.25 & 13.0 \\
\hline $10^{-4}$ & 84.4 & 41.22 & 61.00 & 29.27 \\
\hline \multicolumn{5}{|c|}{$N=25$, near-field electrostatic interactions } \\
\hline$\infty$ & 0.11 & 0.11 & 0.0 & 0.0 \\
\hline $10^{-1}$ & 0.16 & 0.15 & 0.04 & 0.09 \\
\hline $10^{-2}$ & 1.09 & 0.96 & 0.37 & 0.41 \\
\hline $10^{-3}$ & 20.10 & 18.40 & 4.44 & 3.52 \\
\hline $2 \times 10^{-4}$ & 29.79 & 22.00 & 23.20 & 13.75 \\
\hline $10^{-4}$ & 60.79 & 52.46 & 23.92 & 16.72 \\
\hline \multicolumn{5}{|c|}{$N=49$, no near-field electrostatic interactions } \\
\hline $10^{-2}$ & 1.30 & 1.55 & 0.98 & 1.83 \\
\hline $10^{-3}$ & 19.00 & 10.50 & 6.88 & 5.19 \\
\hline \multicolumn{5}{|c|}{$N=50$ (rectangular box), no near-field electrostatic interactions } \\
\hline $10^{-2}$ & 1.22 & 1.20 & 0.63 & 0.14 \\
\hline $10^{-3}$ & 15.62 & 15.17 & 6.81 & 2.39 \\
\hline $10^{-4}$ & 58.18 & 14.27 & 40.15 & 53.13 \\
\hline
\end{tabular}

bution to the total viscosity steadily increases and eventually dominates at $M a=2 \times 10^{-4}$. The difference in the effective viscosities computed with and without the near-field electrostatic interactions is only about $20 \%$ at the smallest $M a$ of $10^{-4}$. At this $M a$, the hydrodynamic viscosity is about the same independent of the addition of near-field electrostatic interactions, so the entire increase is due to the increased electrostatic interactions and not to any fundamental changes to the microstructure. This rather small difference is due to the modest dielectric constant ratio used here, which is typical of current ER fluids. It is expected that for higher dielectric constant ratios, the addition of the nearfield interactions would become more important, especially since the interactions are singular for $\epsilon_{p} / \epsilon=\infty$. The importance of the dielectric constant ratio on the rheology is examined in greater detail in a future paper. ${ }^{21}$

The total viscosities are converted into the shear stresses in Fig. 6 by multiplying them by their corresponding $M a$. This shear-stress shear-rate plot has the asymptotic features of the Bingham plastic model for the shear stress. The shear stress approaches a constant value or Bingham yield stress at small $M a$ or shear rates. At high $M a$ the viscosity increases linearly as a Newtonian fluid. Here the shear stress is nondimensionalized by $2 \epsilon(\beta E)^{2}$, and we see the Bingham yield stress scales with the square of the electric ficld as observed by $\mathrm{Uejima}^{8}$ and Marshall et al. ${ }^{9}$

Although the shear-stress shear-rate plot has the asymptotic features of a Bingham plastic, the Bingham shear-stress model does not match the rheological data well for all $M a$. The Bingham model plotted in Fig. 6 assumes a yield stress of 0.10 and a plastic viscosity of 2.85 , the infinite $M a$ limit of the viscosity. This model underestimates the 


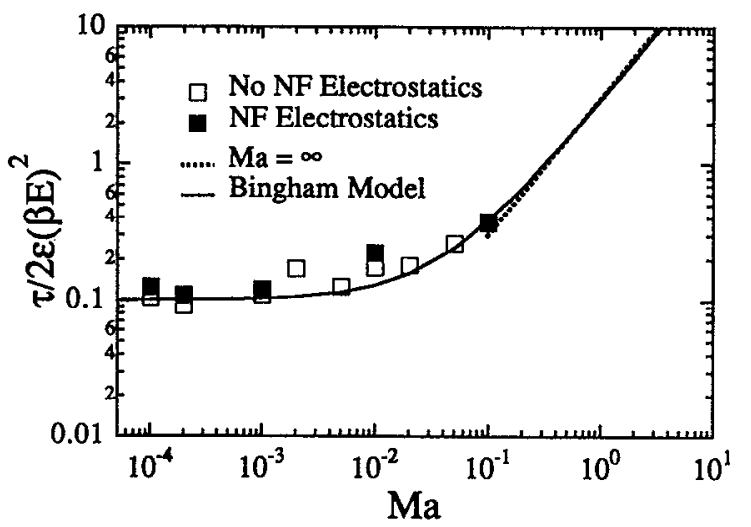

FIG. 6. Shear stress for the monolayer simulations. The shear stress is normalized by electrostatic forces per unit area rather than the viscous forces. The solid line is a Bingham shear stress model assuming a constant plastic viscosity. The NF indicates near-field electrostatic interactions were included.

shear stress in the intermediate $M a$ region of $10^{-1}$ to $10^{-3}$. From the data in Fig. 6, the ER stress $\left(\eta^{\mathrm{ER}} M a\right)$ is effectively the dynamic yield stress as $M a$ vanishes, and the hydrodynamic viscosity is the plastic viscosity. This plastic viscosity, however, is not independent of $M a$. It has two different plateau values at the extremes and is shear thinning in the intermediate region. The value of the plastic viscosity is inconsequential at low $M a$ where the dynamic yield stress dominates the stress, and, at high $M a$, the hydrodynamic viscosity times the shear rate dominates the stress, so the stress at the extremes is modeled well by a Bingham shear-stress model with the aforementioned parameters. However, since the plastic viscosity shear thins in the intermediate region, the true plastic viscosity of the Bingham model actually increases in the intermediate region, increasing the viscosity compared to the Bingham model. The actual shear-stress equation should be

$$
\tau=\tau_{B}+\eta_{B}(\dot{\gamma}) \dot{\gamma}
$$

to take account of the varying behavior of the plastic viscosity.

The simulations of Klingenberg et al. ${ }^{16}$ for a monolayer at an areal fraction of 0.4 predict a dynamic yield stress of 0.014 versus our prediction of 0.12 . Their lower yield stress is probably due to their exclusion of many-body and near-field electrostatic interactions which increase the electrostatic forces. Their simulations with the ad hoc addition of twobody forces in Ref. 17 were not done for the conditions presented here, so no comparison can be made.

Figures 7-9 are time traces of the four different viscosities of the suspension at $M a=\infty, 10^{-2}$, and $10^{-4}$. The heavy dotted lines are the average or running average viscosity up to that particular time. For all but the last $M a$, nearfield electrostatic interactions are not included. The time traces are actually qualitatively similar at identical $M a$ irrespective of the addition of near-field electrostatic interparticle forces. For $M a \geqslant 10^{-3}$, the initial particle configuration was randomly selected by Monte Carlo methods. For the smaller $M a$ the configuration in Fig. 4(b) was the initial position of the particles.

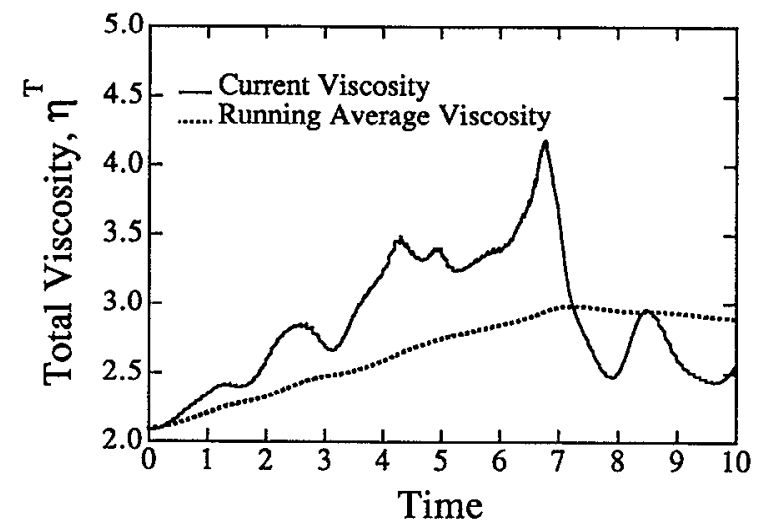

FIG. 7. Time trace for total viscosity for $N=25$ and $M a=\infty$ (no electric field). Both the current and running average effective viscosity are illustrated.

For the infinite $M a$ there is only the hydrodynamic viscosity as seen in Fig. 7. The viscosity slowly increases and mildly fluctuates about its mean of 2.85. It takes about 7 time units to reach this steady state as the initial random microstructure is distorted into the shear configuration. As the $M a$ decreases, the magnitude of the four viscosities increases. The fluctuations of the four viscosities increase dramatically as well. These large excursions are mostly due to large excursions in the hydrodynamic viscosity, but there are also large variations in the other viscosities at these times as well. The time to reach steady state appears to be much smaller for the lower $M a$ and is about 3 or 4 time units.

The hydrodynamic viscosity $\eta^{H}$ is, of course, always positive, but the particle viscosity $\eta^{s^{P}}$ is mostly negative as expected from the discussion in Sec. II B. The $\mathbf{x F}^{\mathbf{P}}$ viscosity $\eta^{X F}$ is mostly positive, although it too is negative sometimes, particularly near the rapid fluctuations. The net or total viscosity is positive for most of the time. These occasionally negative total viscosities, $\eta^{T}$, and large excursions reflect the limitation of the finite number of particles in the simulation. A real system would sample several thousand particles and the net fluctuations would be much smaller. The average values, however, are still reasonable, especially in light of the forthcoming comparison to experimental results.

To best interpret these time traces, we must examine the microstructure of the suspension. A video animation of the dynamic simulations is the best visual aid to see how the microstructure changes with $M a$ and to correlate the motion of the particles to the viscosities. Such a video animation has been made in conjunction with Dr. Andrew Kraynik at the Sandia National Laboratories and has been extremely helpful in understanding ER fluids. Here we present only selected snapshots of the suspension microstructure to describe the dynamics of the suspension and relate it to the rheology, although we shall refer to observations from the video as well. In the snapshots, the average electric field extends from the bottom to the top of the page and the shear flow is from left to right. Again, the outlined box is the periodic cell which is sometimes distorted due to the shcaring motion. In 


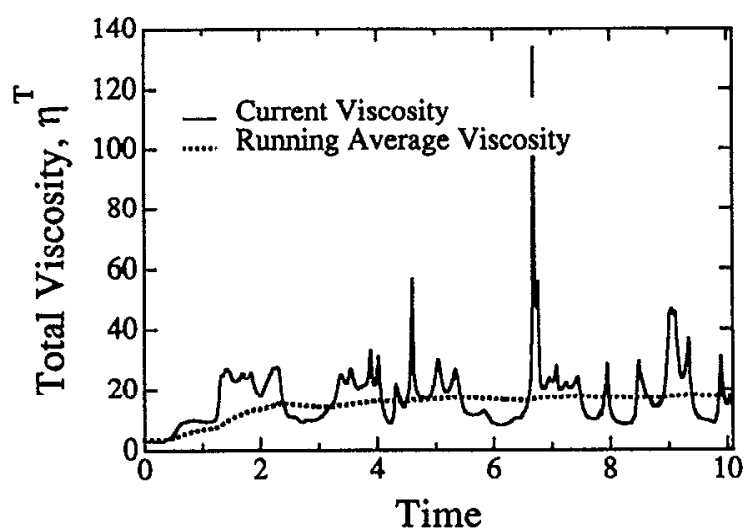

(a)

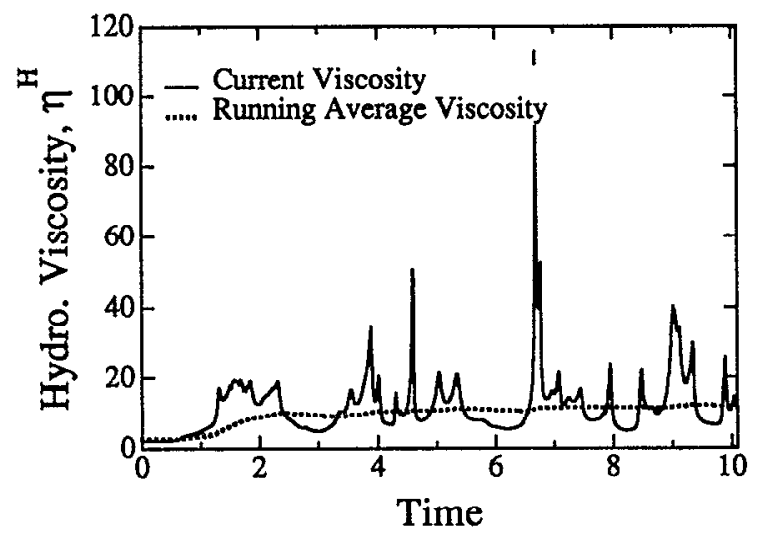

(b)

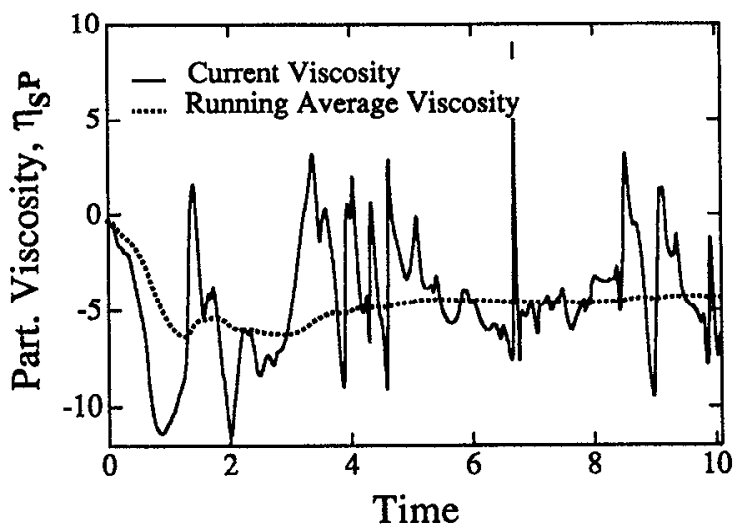

(c)

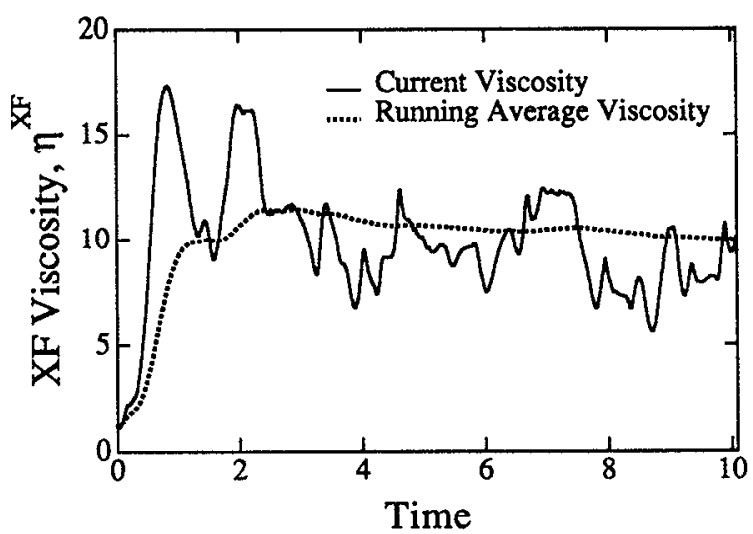

(d)

FIG. 8. Time traces of the (a) total, (b) the hydrodynamic, (c) the particle, and the (d) $x F^{\mathbf{P}}$ viscosity for $N=25$ and $M a=10^{-2}$.

the simulations the periodic cell is reset at a strain or, equivalently, a time of unity.

For $M a=\infty$, the suspension has relatively little structure compared to those with an applied electric field. The particles are fairly randomly dispersed throughout the periodic cell. The small amount of structure, readily apparent from the video animation, has particles driven toward each other along the axis of compression of the shear flow and away from each other along the axis of extension of the flow. The suspension effective viscosity is about 2.85 .

Figure 10 are snapshots of the microstructure for $M a=10^{-2}$. At $t=1.4$, we see the chain structure roughly aligned with the electric field. It is slightly distorted off the axis of the electric field due to the shear flow. At this point the total viscosity is about 25 and, as seen in Fig. 8, is mostly due to the hydrodynamic viscosity of about 20 , with the total ER contribution of about 5. Based upon the rheology, the hydrodynamic forces are still substantial compared to the electrostatic forces despite the rather small $M a$. The electrostatics, however, have altered the microstructure by forming percolating clusters that span the periodic cell. These percolating structures increase the hydrodynamic viscosity. Since the particles are perfectly rigid, they are extremely good con- ductors of stress. Since viscosity can be interpreted as the ability to transmit stress across a velocity gradient, it is not surprising that the hydrodynamic viscosity, which measures this contribution, is large when there are the efficient stress transmission paths provided by the percolating clusters. The hydrodynamic viscosity excursions seen in all the time traces are, in fact, correlated to the formation of these percolating structures with the particles in very close contact. The lubrication forces that prevent overlap also generate extremely large stresses for particles near contact and in relative motion. These stresses increase with decreasing particle spacing which decreases with decreasing $M a$.

Probably the most dramatic change in the microstructure occurs at $M a \leqslant 2 \times 10^{-4}$. At these $M a$ there are two distinct kinds of deformation of the suspension microstructure. For initially aligned chains the suspension is rigid and appears to deform like an elastic body. Figures $11(\mathrm{a})-11(\mathrm{~b})$ show the configuration of the suspension for $M a=10^{-4}$ at $t=4.5$ and 4.6. The motion of the chains is almost affine. As the deformation continues, the effective viscosity increases because of the increasing $\eta^{X F}$ due to the straining of the chains as seen in Fig. 9(d). At a critical strain, occurring at about $t=4.95$, there is a second type of motion. The chain 


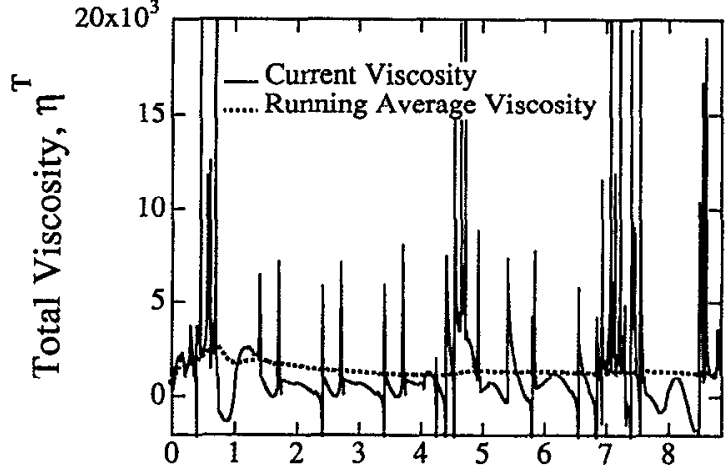

(a)

Time

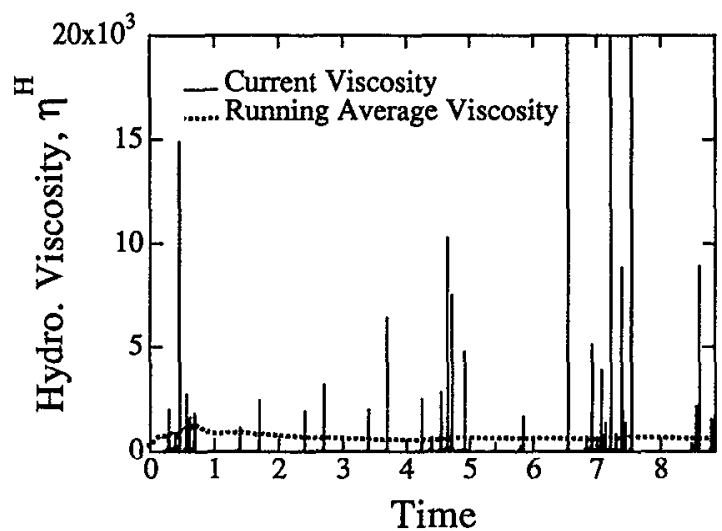

(b)

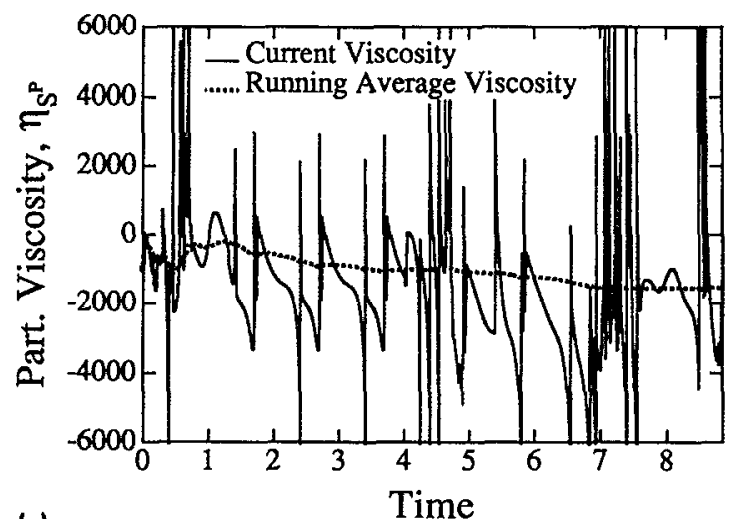

(c)

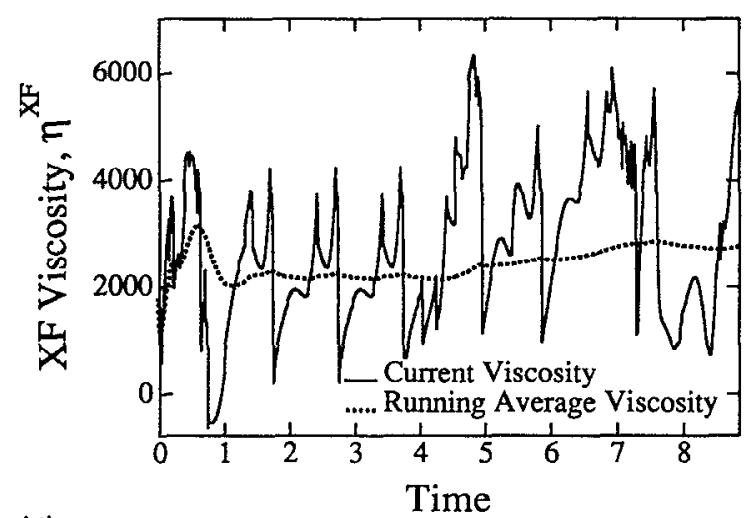

(d)

FIG. 9. Time traces of the (a) total, (b) the hydrodynamic, (c) the particle, and the (d) $x F^{\mathrm{p}}$ viscosity for $N=25^{2}$ and $M a=10^{-4}$.

breaks in half or "snaps" in two and rapidly translates through the fluid until it runs into its periodic image as shown in Figs. $11(\mathrm{c})-11(\mathrm{~d})$ at times $t=4.95$ and 5.00. This time scale is much shorter than that of the inverse shear rate and is, in fact, proportional to $\dot{\gamma}^{-1} M a$ since it is motion driven by the electrostatic forces. Note also that $\eta^{X F}$ has dropped dramatically since the chain has gone from a highly strained state to an almost aligned state. The other viscosities also fluctuate dramatically during this rapid reconfiguration. At $t=4.95, \eta^{H}$ increases because now the particles at the breaking point can move rapidly past one another in a shearlike motion unlike the elastic-body-like deformation during which relative motion was impossible since the particles were all packed tightly together. The hydrodynamic viscosity drops back down when the chain reforms and the particles can again no longer move relative to one another. The $\eta^{S^{P}}$ also changes and actually increases and decreases rapidly at this point. The increases in the particle stress at this point can be attributed to the rapid translation of the chain counter the bulk shear flow which resists the bulk motion.

This interchain rearrangement is actually one of three types of rapid microstructural rearrangements observed in the simulation of the ER fluids at small $M a$. The other two, both preceded by the slow elastic-body-like deformation, are intrachain rearrangements and network rearrangements. The intrachain rearrangement is depicted in Figs. 12(a)12 (b) for $M a=10^{-4}$. Between $t=6.55$ and 6.60 the chain

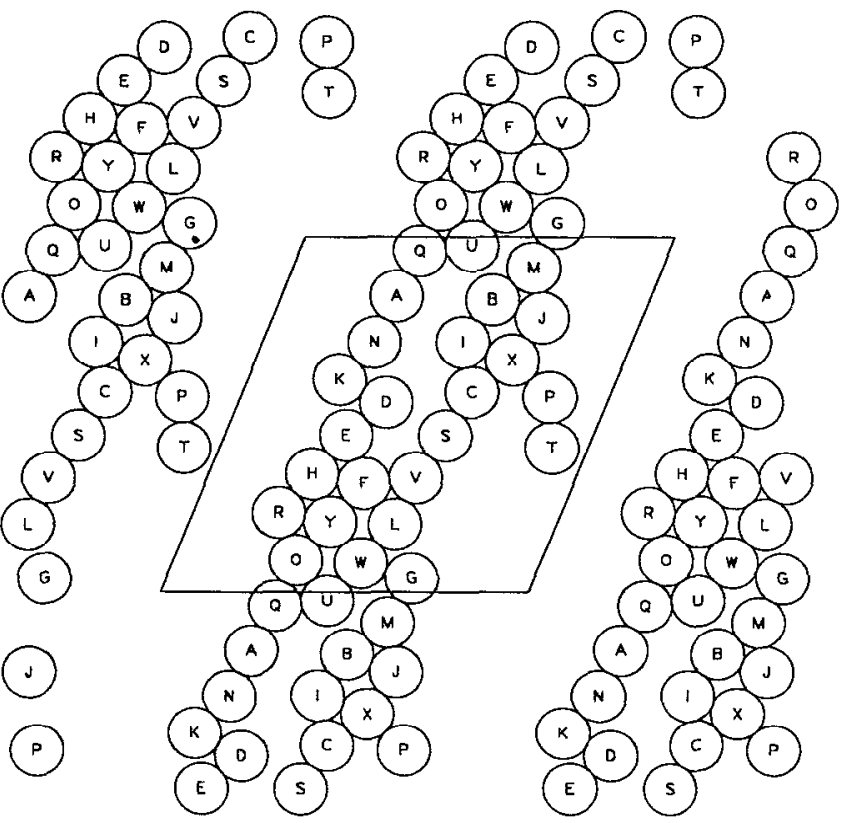

FIG. 10. Instantaneous snapshot of the suspension microstructre for the flowing system at $M a=10^{2}$ for $t=1.4$. The original starting configuration was generated by Monte Carlo methods. Note the percolating chain of particles in sharp contrast to the relatively structureless flow with no electric field. The outlined rhombus is the strained periodic cell and is reset to a square at a strain of unity. 


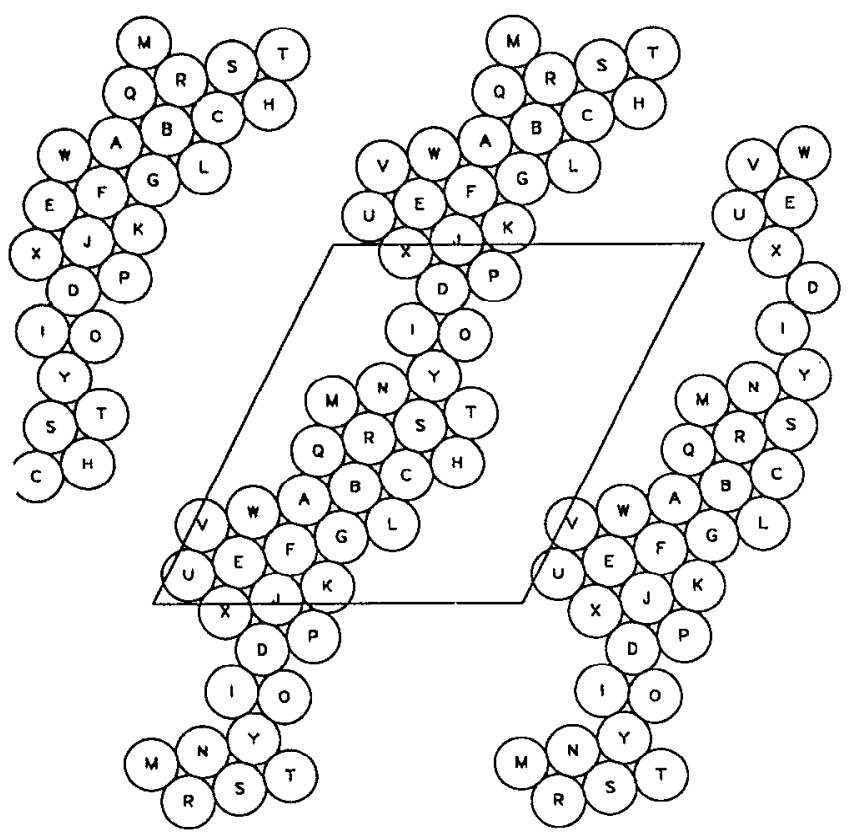

(a)

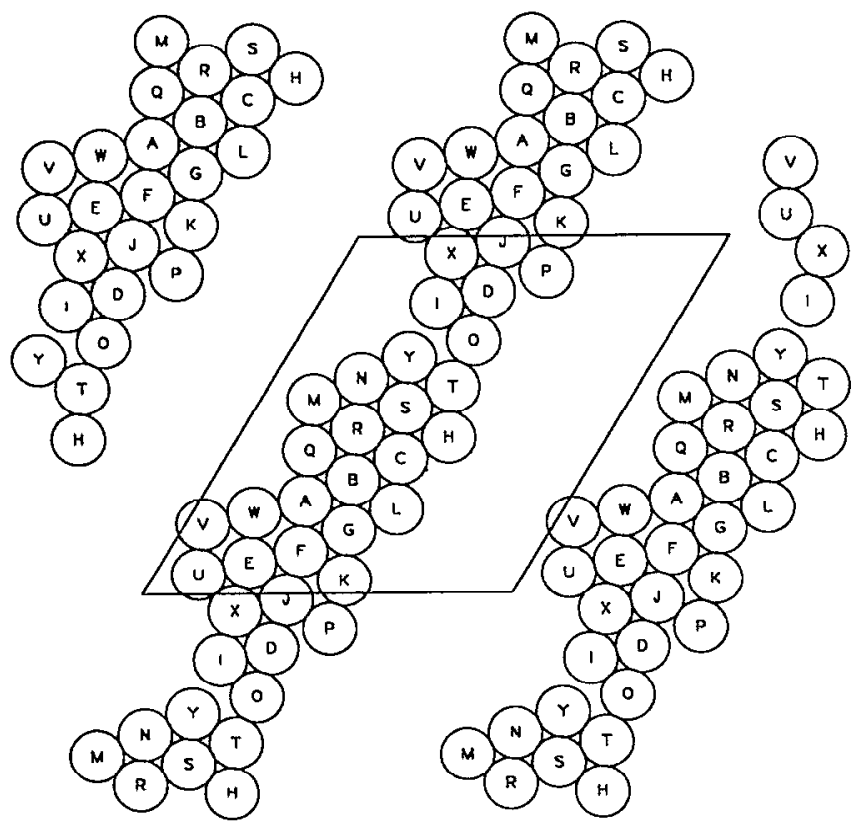

(b)
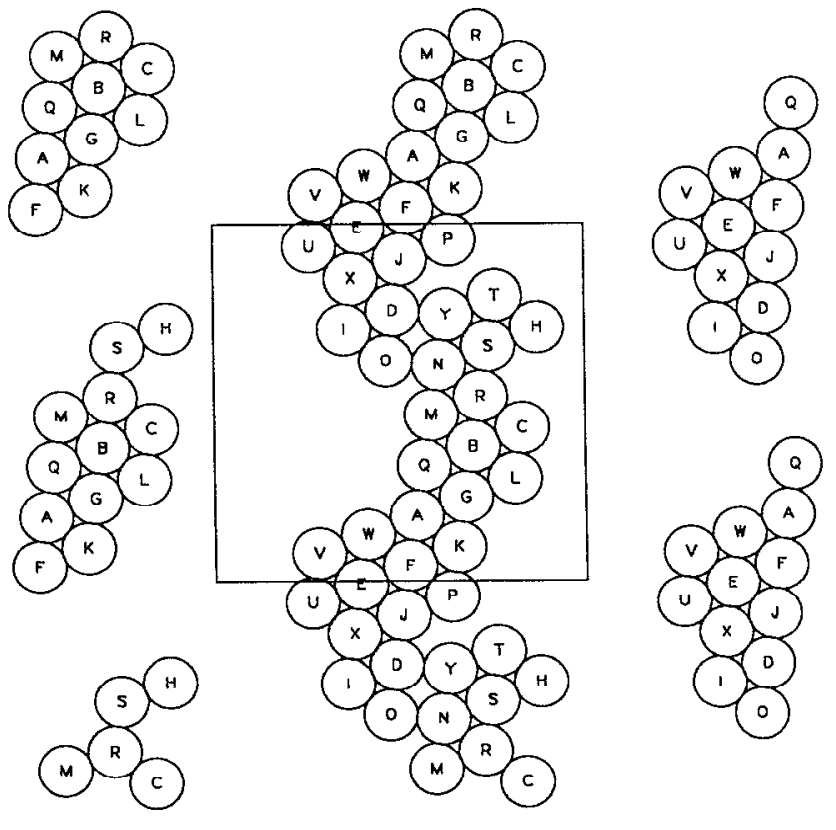

(d)

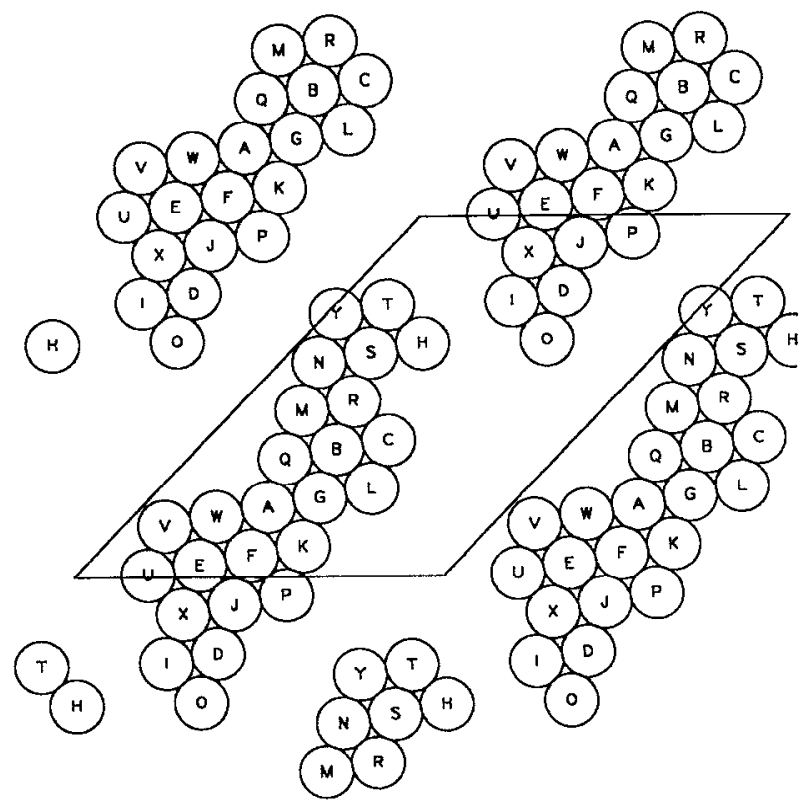

(c)

FIG. 11. Instantaneous snapshots for $N=25$ and $M a=10^{-4}$. This sequence of configurations illustrates rapid interchain microstructural rearrangements. The original starting configuration is illustrated in Fig. 5 (b). (a) $t=4.5$; (b) $t=4.6$; (c) $t=4.95$; (d) $t=5.0$. Note the chain strained slowly until $t=4.95$ at which point it snapped and rapidly reformed with its periodic image.

rapidly jumps over so now particles $C$ and $Q$ are in contact. The $\eta^{X F}$ has also dropped precipitously at this point. Note that there is also rapid $\delta$-function-like increases of the other viscosities. The network rearrangement is illustrated by the sequence of snapshots in Figs. 13(a) and 13(b), again at $M a=10^{-4}$. The microstructure is strained to the point where it forms a network illustrated in Fig. 13(a) at 


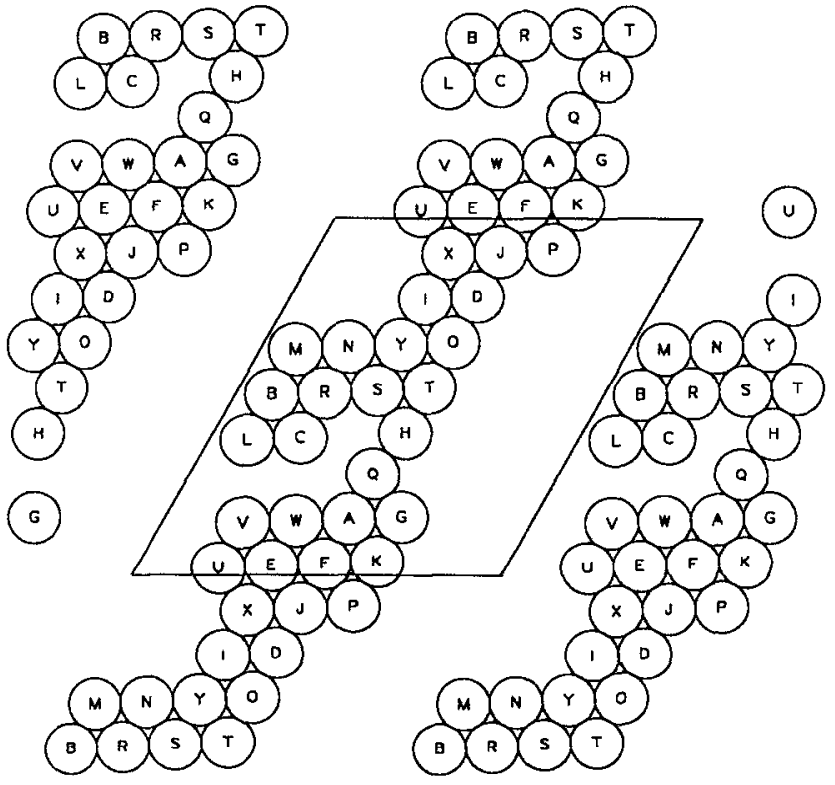

(a)

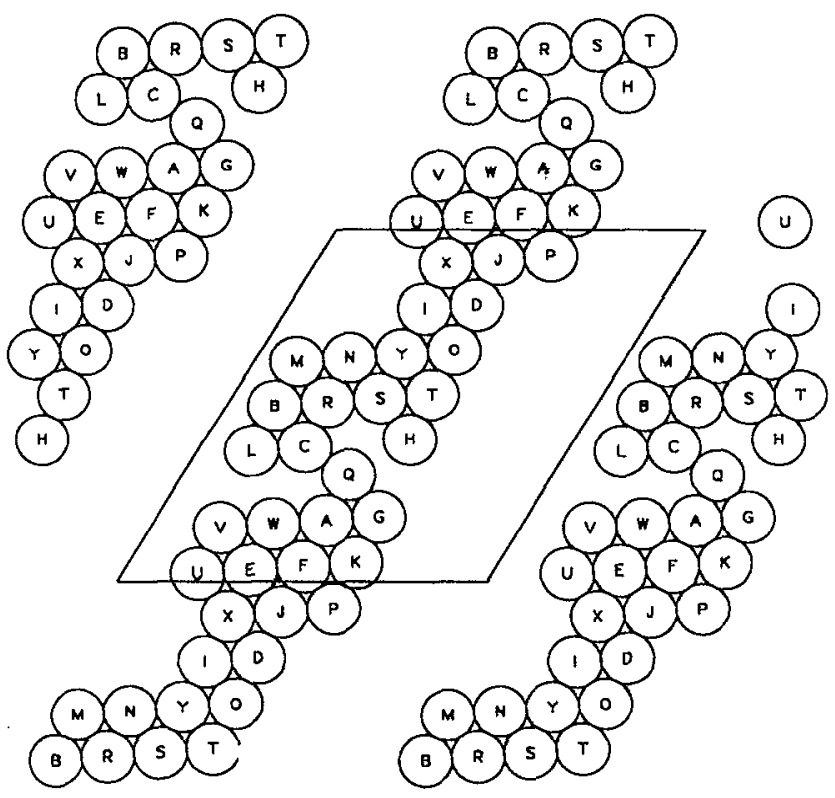

(b)

FIG. 12. Instantaneous snapshots for $N=25$ and $M a=10^{-4}$. This sequence of configurations illustrates rapid intrachain microstructural rearrangements. (a) $t=6.55$ and (b) $t=6.6$. Note the rapid jump made by particle $C$ to $Q$.

ment-all have similar manifestations in the instantaneous viscosities. From here on out all three rearrangements will be sometimes referred to as "snapping" of the microstructure.

Although not readily apparent from the time traces of the viscosities for 25 particles (but from the video animations), these rapid reconfigurations are not dramatic (i.e., not very fast ) until about $M a \leqslant 2 \times 10^{-4}$, which is about the $M a$ where the hydrodynamic viscosity plateaus. Similar microstructural reconfigurations as shown in Figs. 11-13 are

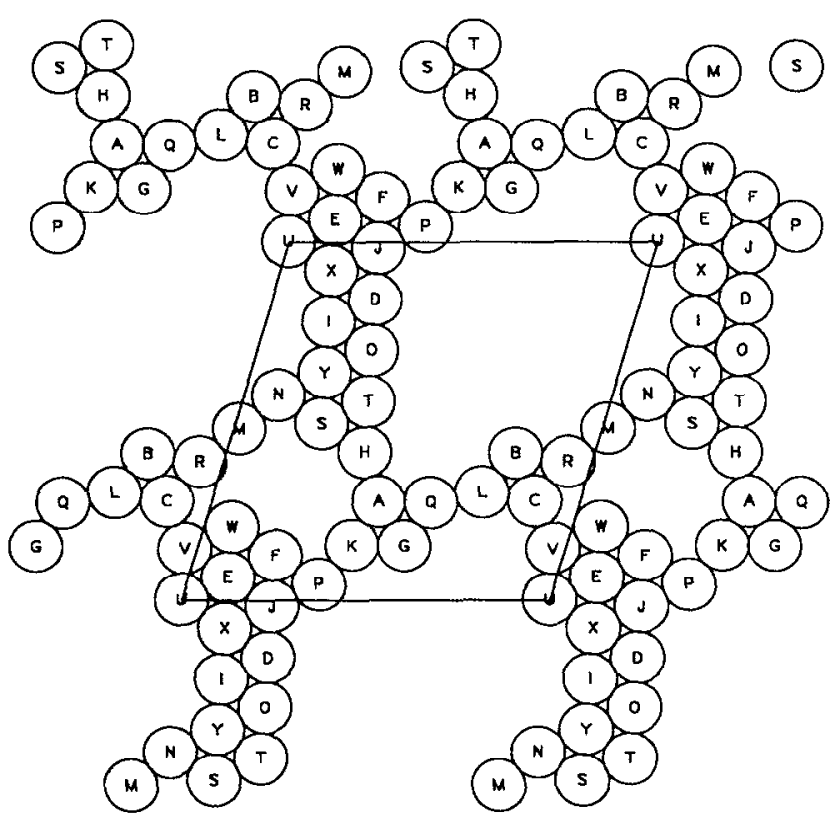

(a)

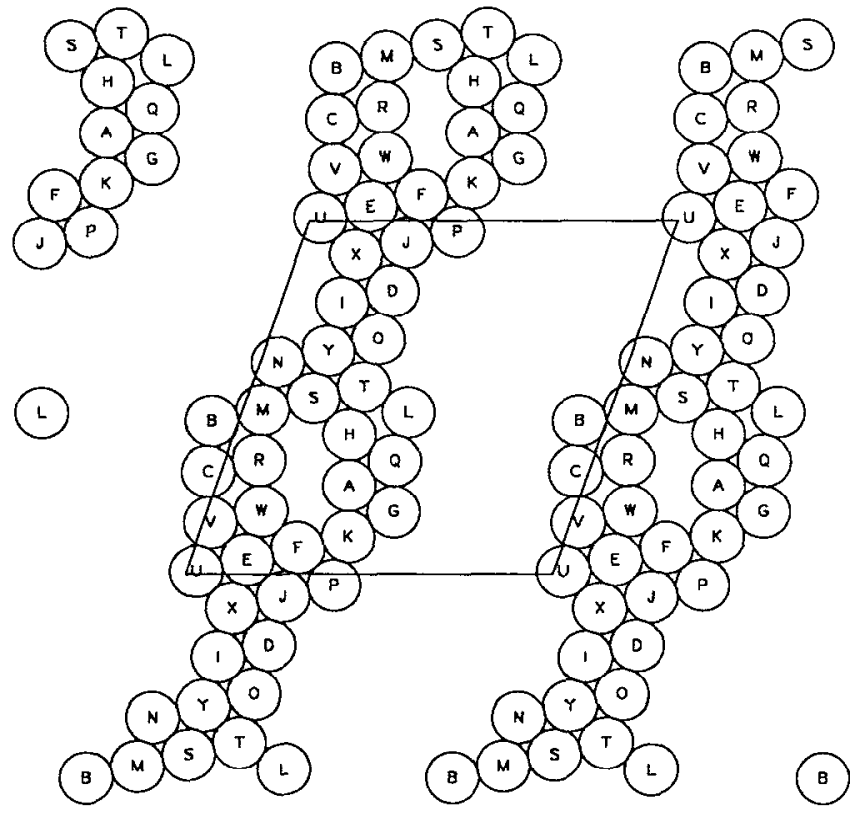

(b)

FIG. 13. Instantaneous snapshots for $N=25$ and $M a=10^{-4}$. (a) $t=7.30$, note the formation of an interconnecting network; (b) $t=7.35$, note the rapid contraction of the network into percolating chains. This sequence of configurations illustrates rapid network rearrangements.

also seen at $M a=2 \times 10^{-4}$. Since the average microstructure is here independent of $M a$, the average hydrodynamic viscosity is also independent of $M a$ as observed by its upper plateau value. Thus the shear thinning of the hydrodynamic or plastic viscosity at intermediate $M a$ is the shear induced breakdown or melting of the strongly chained microstructure for low $M a$.

We also note here that the slow straining and rapid reconfigurations are exceptionally dramatic and well por- 
trayed in the video animations of nine particles. This is due to the fact that nine particles form shorter chains that translate more quickly when they break in half. The effect of chain length on the time scale for the snapping of the microstructure might explain why such a low $M a$ is needed to observe an ER response, i.e., viscosity growing as the square of the electric field. The $M a$ number is a ratio of the viscous forces to the electrostatic forces, and the length scale for characterizing the hydrodynamic resistance is the radius of a sphere making up the particle chains. The hydrodynamic resistance perhaps should be characterized by some function appropriate for a rodlike chain that increases with the length of the chain and its aspect ratio. This will be, of course, greater than the sphere radius. This new $M a$ would be much larger and closer to the $O(1)$ value expected at the transition point rather than the $O(0.01)$ value observed in Fig. 5 .

Finally, it is worthwhile to examine the electrostatic energy of the suspension $\mathscr{U}$ for the different $M a$. Figure 14 illustrates the time trace of the energy for $M a=10^{-2}$ and $10^{-4}$. For the larger $M a$ number the energy rises and falls in a rather smooth fashion. For the smaller $M a$ numbers, the energy drops are quite dramatic. In general as $M a$ decreases, the energy drops more rapidly after slowly increasing. In fact the time trace of the energy is probably the best lumped rep-

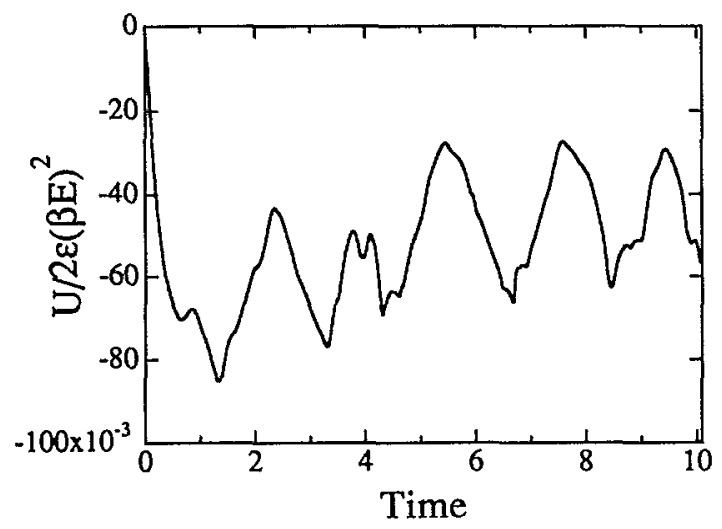

(a)

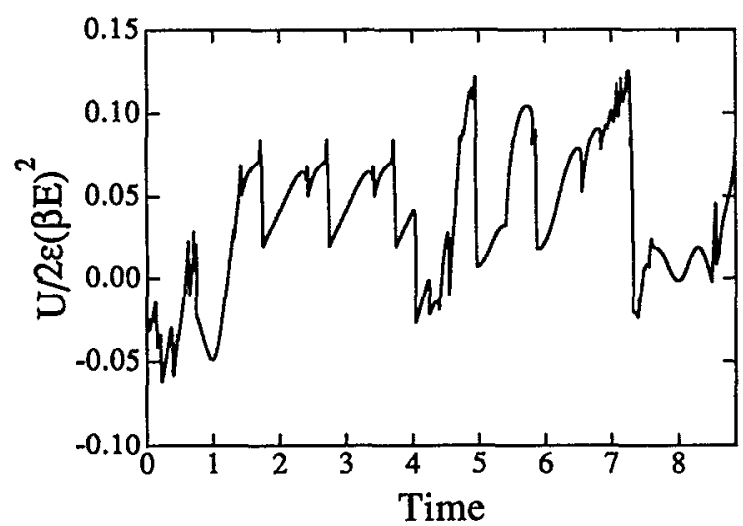

(b)

FIG. 14. Time traces of the electrostatic energy for (a) $M a=10^{-2}$; (b) $M a=10^{-4}$. resentation of the dynamics of the microstructure at low $M a$. The slowly increasing energy of the system corresponds to the relatively slow elastic-body-like deformation, and the precipitous drop in the electrostatic energy occurs when the microstructure rapidly reconfigures or snaps. This is clear from comparison of the microstructures in Figs. 11-13 to the time trace in Fig. 14(b). The connection between the macroscopic energy and the microstructure's dynamics implies that energy is being stored during the elastic-body-like deformation and viscously dissipated during the snapping process.

The behavior of the microstructure and the energy also makes us suspect that the energy jumps and the Bingham or dynamic yield stress are related. The rapid reconfigurations and the dominance of the ER viscosity or stress on the rheology both occur for $M a \leqslant 2 \times 10^{-4}$. The yield stress, although often interpreted as a force per unit area, is also an energy per unit volume as is $\mathscr{U}$. Furthermore, both the yield stress and the energy jumps scale as $2 \epsilon(\beta E)^{2}$. At this point it is only supposition, but in our future paper ${ }^{21}$ we show through a total energy balance that indeed the yield stress is proportional to the energy jumps and the proportionality is given by the frequency of the snapping of the microstructure.

\section{Dynamic simulation results: First normal stress difference}

In addition to the shear viscosity, the first normal stress difference of the suspension can be computed from the bulk stress. Like the shear viscosity, the total first normal stress difference, $\chi^{T}$ is given by

$$
\chi^{T}=\chi^{H}+\chi^{\mathrm{ER}},
$$

where

$$
\chi^{T}=\left\langle\Sigma_{x x}\right\rangle-\left\langle\Sigma_{y y}\right\rangle
$$

so

$$
\begin{aligned}
\chi^{H}= & \frac{5}{3} \phi_{A} \frac{1}{N} \sum_{\alpha=1}^{N}\left[\left(\mathbf{S}_{\alpha}^{H}\right)_{x x}-\left(\mathbf{S}_{\alpha}^{H}\right)_{y y}\right], \\
\chi^{\mathrm{ER}}= & \frac{5}{3} \phi_{A} \frac{1}{M a N} \sum_{\alpha=1}^{N}\left[\left(\mathbf{S}_{\alpha}^{p}\right)_{x x}-\left(\mathbf{S}_{\alpha}^{p}\right)_{y y}\right] \\
& -\frac{5}{3} \phi_{A} \frac{1}{M a 2 N} \sum_{\alpha}^{N} \sum_{\beta}^{N}\left\{\left[\left(\mathbf{x}_{\alpha}-\mathbf{x}_{\beta}\right) \mathrm{f}^{\alpha \beta}\right]_{x x}\right. \\
& \left.-\left[\left(\mathbf{x}_{\alpha}-\mathbf{x}_{\beta}\right) \mathrm{f}^{\alpha \beta}\right]_{y y}\right\} .
\end{aligned}
$$

The hydrodynamic normal stress difference is $\chi^{H}$ and the particle stress and thermodynamic contributions have been lumped into a single ER normal stress difference $\chi^{\mathrm{ER}}$. Since the electrostatic forces in the $z$ direction are not needed for the dynamic simulation, they are not computed, so the second normal stress difference is not known. The first normal stress differences for different $M a$ are listed in Table IV and plotted in Fig. 15. Note that the normal stress difference is nondimensionalized by $\eta \dot{\gamma}$. The first normal stress difference is practically zero for no electric field, as expected for a hardsphere suspension of force-free particles. Indeed, in the pure hydrodynamic limit, the normal stress differences must be zero, a consequence of flow reversal symmetry. The small nonzero values are simulation fluctuations. As $M a$ de- 
TABLE IV. Dynamic simulation first normal stress difference. Notc the numbers following the \pm are the $95 \%$ confidence limits of the first normal stress difference. The times of the simulations are identical to the equivalent Ma viscosity data in Table II.

\begin{tabular}{|c|c|c|c|}
\hline$M a$ & $\chi^{T}$ & & $\chi^{\mathrm{ER}}$ \\
\hline \multicolumn{4}{|c|}{$N=25$, no near-field electrostatic interactions } \\
\hline$\infty$ & $0.03 \pm 0.02$ & $0.03 \pm 0.02$ & $0.0 \pm 0.0$ \\
\hline $10^{-1}$ & $-0.09 \pm 0.05$ & $0.04 \pm 0.04$ & $-0.13 \pm 0.06$ \\
\hline $2 \times 10^{-2}$ & $-3.36 \pm 0.21$ & $1.35 \pm 0.18$ & $-4.71 \pm 0.14$ \\
\hline $10^{-2}$ & $-5.42 \pm 0.71$ & $3.58 \pm 0.53$ & $-9.00 \pm 0.36$ \\
\hline $2 \times 10^{-3}$ & $-29.70 \pm 6.17$ & $21.22 \pm 5.58$ & $-50.92 \pm 2.14$ \\
\hline $10^{-3}$ & $-4.95 \pm 15.30$ & $64.20 \pm 14.72$ & $-69.15 \pm 8.01$ \\
\hline $2 \times 10^{-4}$ & $-164 \pm 79.2$ & $133 \pm 50.6$ & $-297 \pm 52.2$ \\
\hline $10^{-4}$ & $-535 \pm 115$ & $87.4 \pm 30.7$ & $-622 \pm 119$ \\
\hline \multicolumn{4}{|c|}{$N=25$, near-field electrostatic interactions } \\
\hline $10^{-2}$ & $-10.80 \pm 2.97$ & $1.44+2.92$ & $-12.24+0.36$ \\
\hline $10^{-3}$ & $-11.76+11.48$ & $15.30+7.93$ & $-27.06+9.87$ \\
\hline $2 \times 10^{-4}$ & $-250 \pm 61.3$ & $158 \pm 33.4$ & $-408 \pm 51.2$ \\
\hline $10^{-4}$ & $-352 \pm 62.0$ & $78.30 \pm 23.90$ & $-430 \pm 58.5$ \\
\hline
\end{tabular}

creases, $\chi^{T}$ becomes increasingly negative and, in fact, grows in magnitude with the square of the electric field or as $\mathrm{Ma}^{-1}$. The $\chi^{H}$ is, on average, positive while the $\chi^{\mathrm{ER}}$ is, on average, negative. The existence of the first normal difference is the direct result of the anisotropic character of an ER fluid.

This negative normal stress difference is not surprising in light of the strong attractive forces holding the chains together. The normal stress due to the electrostatic forces in the $x$ (flow) direction is practically zero since the chains are far apart. The normal stress due to electrostatics in the $y$ direction is very large since the particles in the chains are attracted to one another along the $y$ axis. Thus the difference between the $x x$ and $y y$ normal stresses, $\chi^{\mathrm{ER}}$, is negative, and since they are driven by the electrostatic forces, they scale with the square of the electric field. The positive $\chi^{H}$ is due to the lubricating film pressures countering the motion of the particles. The $\chi^{\mathrm{ER}}$ normal stress difference, however, dominates. Although the first normal stress difference can vary over several orders of magnitude for an ER fluid, the actual

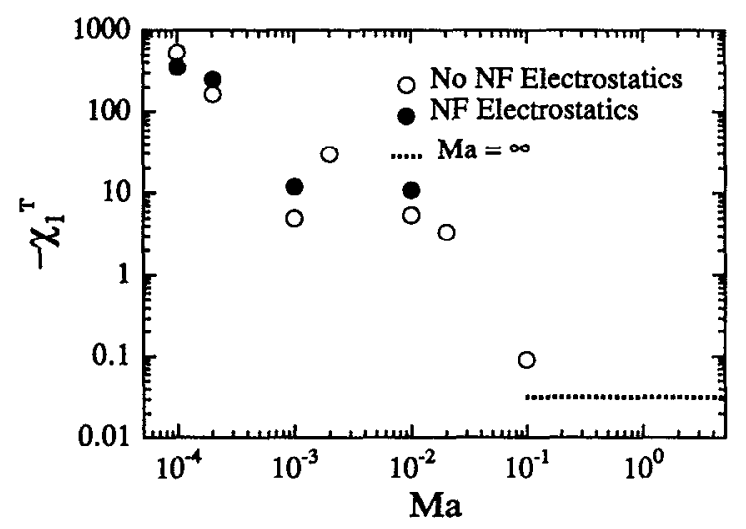

FIG. 15. The negative of the first normal stress difference, $\Sigma_{x x}-\Sigma_{y y}$, nondimensionalized by $\eta \dot{\gamma}$ '. Simulation results with and without the addition of near-field electrostatics are illustrated. The $M a=\infty$ result is practically zero especially considering the fluctuations reported in Table IV. mechanical requirements are quite modest, so normal stress differences are probably not key design considerations.

From Table IV note that the fluctuations are extremely large for small $M a$. Like the effective viscosities, these dramatic fluctuations at $M a=10^{-4}$ are due to the rapid microstructural reconfigurations. At the larger $M a$, the fluctuations are less dramatic since the electrostatic forces are not so large compared to the hydrodynamic forces.

\section{E. Comparison to experimental results}

We compare the simulation effective viscosity to the experimental results of Marshall, Goodwin, and Zukoski. ${ }^{9}$ Their ER fluid consists of hydrated poly (methacrylate) particles in a chlorinated hydrocarbon oil, and the effective viscosities were measured over a large range of $M a$ numbers. To compare the simulation results to their experimental results, the monolayer areal fraction is converted to a volume fraction by multiplying $\phi_{A}$ by two-thirds. This "two-thirds" rule is correct in the dilute limit where the Einstein viscosity correction is $\frac{5}{2} \phi$ in three dimensions and $\frac{5}{3} \phi_{A}$ in a monolayer. It is approximately correct in the limit of closest packing where two-thirds of the maximum packing areal fraction of a two-dimensional lattice is very close to the maximum packing volume fraction for the three-dimensional analog lattice. For a two-dimensional square lattice, for example, the maximum areal fraction is $\pi / 4$, and the maximum volume fraction for the simple cubic lattice is $\pi / 6$. Here the ratio of maximum areal fraction to volume fraction is exactly twothirds. The simulation data then corresponds to an approximate volume fraction of $\phi=0.27$. The closest volume fraction from the experimental data reported was $\phi=0.23$.

Figure 16 compares the simulation results, with and without near-field electrostatic interactions, to the experimental results. The comparison between the two is extremely good at both extremes of the $M a$ number. At the intermediate values of $M a$, the simulation viscosities slightly underpredict the experimental viscosities. The experimental suspensions, even in the absence of the electric field, have a

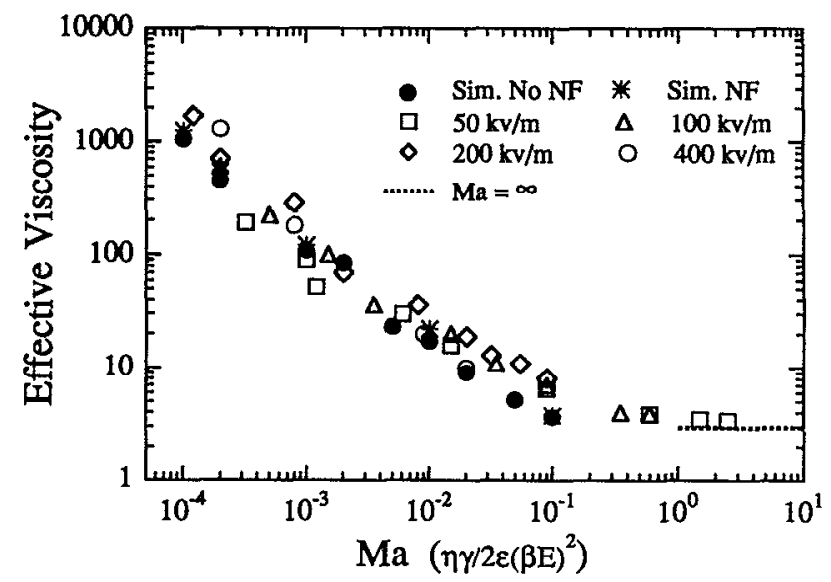

FIG. 16. Comparison of simulation results to the experimental results of Marshall et al. illustrated with the open symbols and the applied field used. The equivalent volume fraction of the monolayer from the "two-thirds" rule is 0.27 and the experimental volume fraction is 0.23 . 
yield stress at low shear rates due to some other interparticle colloidal forces. At low $M a$, where the electrostatic forces dominate, these colloidal forces are unimportant. At the intermediate $M a$ numbers, these colloidal forces might be as important as the electrostatic forces in altering the microstructure and changing the hydrodynamic viscosity as well as making their own contribution to the particle and thermodynamic viscosities. In any event, the comparison is exceptionally good and it appears that the simulation captures both the qualitative and quantitative features of an ER suspension.

\section{F. Effects of periodic boundary conditions}

The successful comparison to experimental data is quite satisfying, but we wonder what effect periodic boundary conditions have on the effective viscosities computed with the simulation and why, in spite of them, the results are in such good quantitative agreement. From the video animations the periodic boundary conditions have a clear effect on the evolution of the microstructure. The interchain rearrangement or chain snapping occurs roughly with a period of one time unit or a strain of one periodic cell. When the chain snaps, it connects to its periodic image and the process, roughly speaking, repeats itself. The effective viscosities and the energy time traces also cxhibit a rough periodicity of one or so for each slow deformation and chain snapping episode in the microstructure.

Klingenberg and Zukoski ${ }^{31}$ have experimentally observed the microstructure in an ER suspension by using a microscope and have made observations that are similar to those discussed here. They observed percolating chains of particles spanning the electrode gap, much like the percolating structures spanning the periodic cell. With the shear flow induced by the moving upper electrode, they observed that the chains do deform much like an elastic body until a critical strain at which point the chains snap. The upper broken chain then reconnects with the nearest forward lower half chain and the process repeats itself with a fairly regular frequency. Thus the periodic breaking and snapping of chains observed in the simulation is entirely realistic. The critical strain observed by Klingenberg and Zukoski, however, varied from 0.4 to 0.6 , unlike the critical strain of unity in the simulations. Clearly, the periodic boundary conditions have had an influence on the critical strain and the snapping frequency. We show later ${ }^{21}$ that the exact value of the critical strain is not important for modest conductivity ratios which explains why the comparison between the experiments and the simulation is good despite this difference.

Using periodic boundary conditions without walls also precludes the existence of more than one chain per periodic cell. Initially, distinct chains are brought near one another due to the shear flow, and the chains coalesce because of the chain-chain attraction discussed in Sec. IV B. For 25 particles and $M a=10^{-4}$, the initial configuration in Fig. 4(b) contains three chains, but at time $t=4.6$ there is only a single chain. In experiments the chains are almost permanently stuck to the walls due to the strong electrostatic force between the particles and the perfectly conducting metal plates. Particle and plate roughness may also play a role in ensuring distinct chains in an ER device. Since there are no walls in the simulation, coalescence will always occur.

In using periodic boundary conditions with no walls at low $M a$, we are simulating a single chain surrounded by its periodic images. Evidently this captures the essential physics since there is excellent agreement between simulation and experimental viscosities. This is not surprising since, with the near-field interparticle interactions within the chain and the far-field interactions among the chains, these conditions capture the important features of slow-chain deformation and rapid microstructural rearrangement observed experimentally and which are so important to the ER response. Indeed, the fact that the single chain fills the periodic box actually gives the correct behavior because it allows the chains from one cell to the next to connect with each other and percolate, just as a single chain would span the electrodes in a true experiment. The simulations, thus, should be interpreted as single-chain simulations for low $M a$. At large $M a$, the hydrodynamic forces dominate and the suspension is almost structureless, so periodic boundary conditions are completely reasonable because the system size is large enough.

Several simulations were done with 50 particles in a rectangular periodic cell as high as the 25 particle simulations and twice as long. At the large $M a$ the effective viscosities for the 50 particles is similar to 25 particles, where the electrostatic forces are small and there is little suspension structure. Here the system size is sufficiently large at 25 particles and do not depend on periodic boundary conditions. At the smallest $M a$ of $10^{-4}$, however, the viscosity for 50 particles is about one-half that of 25 particles at the same $M a$. Because of chain coalescence, there is only one chain in the rectangular periodic cell and half the number of chains per unit length in the 50 particle case compared to 25 particles. In the simulations, the 50 particle chains still break at a critical strain of about one, but since their image is twice as far away, they reconnect and begin the straining-snapping process at half the frequency of the 25 particles. As we show in Ref. 21, the effective viscosity at low $M a$ is proportional to the snapping frequency, so the result is not a weakness in periodic boundary conditions, but rather a confirmation of the relationship between the yield stress and the rate that energy is stored and released.

\section{CONCLUSIONS}

We have presented a method for the dynamic simulation of an ER suspension subject to a simultaneous shear flow and orthogonal electric field. The Stokesian dynamics methodology accurately accounts for both the far- and near-field hydrodynamic interactions among the particles due to the electrostatic forces and the bulk shear flow. The conservative electrostatic interparticle forces were computed from a system electrostatic energy that was determined with the capacitance matrix developed earlier ${ }^{19,20}$ for determining the effective conductivity of a suspension. Because the capacitance matrix contains both far- and near-field electrostatic interactions, the electrostatic forces do also. With the complete simulation, the time-evolved motion and the bulk rheology of the ER suspension were determined as a func- 
tion of the $M a$. The effective viscosity was found to scale as $M a^{-1}$ or with the square of the electric field for low $M a$ as observed in experiments. The material behaves approximately as a Bingham plastic except with a shear thinning plastic viscosity for $M a$ between $2 \times 10^{-4}$ and $10^{-1}$. The effective viscosities compared extremely well to experimental data which indicates that the simulations capture the physics both qualitatively as well as quantitatively.

The dynamics of the motion were correlated with rheology and it was found that, for $M a \leqslant 2 \times 10^{-4}$, the microstructure began to exhibit two distinct motions, namely a slow elastic-body-like deformation and a rapid reconfiguration or snapping of the microstructure. This snapping of the microstructure coincides with a precipitous drop of $\eta^{X F}$ and the electrostatic energy of the system. We suppose at this point that the slow strain and rapid snapping episodes are the key to the ER response and Bingham yield stress. How these microstructural changes and the jumps in the electrostatic energy are related to the Bingham or dynamic yield stress are developed in detail in a future paper. ${ }^{21}$ This theory of the dynamic yield stress will be tested using the dynamic simulation described in this paper.

Finally, the methodology for computing the electrostatic energy and interparticle forces is not limited to ER fluids. Any system of electrically or magnetically interacting particles can be accurately modeled with the method outlined here, which might be useful for other molecular-dynamic simulations or statistical theories.

\section{ACKNOWLEDGMENT}

The authors wish to thank Dr. Andrew Kraynik of the Sandia National Laboratories for many useful comments on this work and for the preparation of a video animation that was helpful for the understanding of the simulation results.
${ }^{1}$ Electrorheological fluids. Proceedings of the Second International Conference on ER Fluids, edited by J. D. Carison, A. F. Sprecher, and H. Conrad (Technomic, Lancaster, PA, 1990).

${ }^{2}$ W. M. Winslow, J. Appl. Phys. 20, 1137 (1949).

${ }^{3}$ R. Rosensweig, Ferrohydrodynamics (Cambridge University, Cambridge, UK, 1985).

${ }^{4}$ G. K. Batchelor, J. Fluid Mech. 41, 545 (1970).

${ }^{5}$ R. W. O'Brien, J. Fluid Mech. 91,17 (1979).

${ }^{6}$ D. L. Klass and T. W. Martinek, J. Appl. Phys. 38, 67 (1967).

${ }^{7}$ D. L. Klass and T. W. Martinek, J. Appl. Phys. 38, 75 (1967).

${ }^{8} \mathrm{H}$. Uejima, Jpn. J. Appl. Phys. 11, 319 (1972).

${ }^{9}$ L. Marshall, J. W. Goodwin, and C. F. Zukoski, J. Chem. Soc. Faraday I 85, 2785 (1989).

${ }^{10}$ P. A. Arp, R. T. Foister, and S. G. Mason, Adv. Colloid Interface Sci. 12, $295(1980)$

"D. J. Klingenberg, Ph.D. thesis, University of Illinois, 1990.

${ }^{12}$ Z. Mimouni, G. Bossis, C. Mathis, A. Meunier, and C. Paparoditis, Prog. Colloid Polym. Sci. 81, 120 (1990).

${ }^{13}$ E. Lemaire and G. Bossis, J. Phys. D (to be published).

${ }^{14}$ A. P. Gast and C. F. Zukoski, Adv. Colloid Interface Sci. 30, 153 (1989).

${ }^{15}$ P. M. Adriani and A. P. Gast, Phys. Fluids 31, 2757 (1988).

${ }^{16}$ D. J. Klingenberg, F. van Swol, and C. F. Zukoski, J. Chem. Phys. 94, 6160 (1991).

${ }^{17}$ D. J. Klingenberg, F. van Swol, and C. F. Zukoski, J. Chem. Phys. 91, 6170 (1991).

${ }^{18}$ M. J. Whittle, Non-Newtonian Fluid Mech. 37, 233 (1990).

${ }^{19}$ R. T. Bonnecaze and J. F. Brady, Proc. R. Soc. London, Ser. A 430, 285 (1990).

${ }^{20}$ R. T. Bonnecaze and J. F. Brady, Proc. R. Soc. London, Ser. A 432, 445 (1991).

${ }^{21}$ R. T. Bonnecaze and J. F. Brady, J. Rheology (in press).

${ }^{22}$ J. F. Brady and G. Bossis, Annu. Rev. Fluid Mech. 20, 111 (1988).

${ }^{23}$ L. Durlofsky, J. F. Brady, and G. Bossis, J. Fluid Mech. 180, 21 (1987).

${ }^{24}$ J. F. Brady, R. J. Phillips, J. C. Lester, and G. Bossis, J. Fluid Mech. 195, 257 (1988).

${ }^{25}$ H. Brenner, Chem. Eng. Sci. 19, 631 (1964).

${ }^{26}$ G. K. Batchelor, J. Fluid Mech. 83, 97 (1977).

${ }^{27}$ J. D. Jackson, Classical Electrodynamics, 2nd ed. (Wiley, New York, 1975).

${ }^{28}$ L. D. Landau and E. M. Lifshitz, Electrodynamics of Continuous Media, 2nd ed. (Pergamon, New York, 1984).

${ }^{29}$ D. J. Jeffrey, J. Ind. Math. Appl. 22, 337 (1978).

${ }^{30} \mathrm{H}$. Conrad, M. Fisher, and A. F. Sprecher, Proceedings of the Second International Conference on ER Fluids, edited by J. D. Carlson, A. F. Sprecher, and H. Conrad (Technomic, Lancaster, PA, 1990).

${ }^{31}$ D. J. Klingenberg and C. F. Zukoski, Langmuir 6, 15 (1990). 